\title{
OPTICAL MICROFLUIDIC WAVEGUIDES AND SOLUTION LASERS OF COLLOIDAL SEMICONDUCTOR QUANTUM WELLS
}

\author{
A THESIS SUBMITTED TO \\ THE GRADUATE SCHOOL OF ENGINEERING AND SCIENCE \\ OF BILKENT UNIVERSITY \\ IN PARTIAL FULFILLMENT OF THE REQUIREMENTS FOR \\ THE DEGREE OF \\ MASTER OF SCIENCE \\ IN \\ MATERIALS SCIENCE AND NANOTECHNOLOGY
}

By

Joudi Maskoun

July 2020 
Optical Microfluidic Waveguides and Solution Lasers of Colloidal Semiconductor Quantum Wells

By Joudi Maskoun

July 2020

We certify that we have read this thesis and that in our opinion it is fully adequate, in scope and in quality, as a thesis for the degree of Master of Science.

Hilmi Volkan Demir(Advisor)

Emine Yegân Erdem(Co-Advisor)

Onur Tokel

Talha Erdem

Approved for the Graduate School of Engineering and Science:

Ezhan Karaşan

Director of the Graduate School 


\title{
ABSTRACT \\ OPTICAL MICROFLUIDIC WAVEGUIDES AND SOLUTION LASERS OF COLLOIDAL SEMICONDUCTOR QUANTUM WELLS
}

\author{
Joudi Maskoun \\ M.S. in Materials Science and Nanotechnology \\ Advisor: Hilmi Volkan Demir \\ Co-Advisor: Emine Yegân Erdem
}

July 2020

Microfluidics has become an important technology platform offering many applications including point-of-care systems, lab-on-a-chip (LOC) devices, and drug delivery and separation. For this technology to reach its full potential, many improvements and components are being heavily researched and utilized to help broaden the range of its applications. One such important application is the implementation of lasers in microfluidic networks. Microfluidic lasers are being employed as sensors and light sources for use in chemical and biological reaction promoting and flow cytometry. Microfluidic amplified spontaneous emission (ASE) and lasing using fluorescent dyes embedded in liquid-liquid waveguides has been previously reported. The performance of these devices may be significantly improved using colloidal semiconductor quantum wells, also known as nanoplatelets (NPLs), which possess optical properties desirable for lasing. In this work, different than previous works, optical microfluidic waveguides and solution lasers of NPLs are proposed and demonstrated. To this end, a Fabry-Pérot cavity is created in a microfluidic channel encapsulated with polydimethylsiloxane (PDMS) to achieve in-solution lasing with NPLs. The microfluidic devices are fabricated using soft lithography and implemented as a platform for observing optical gain from NPLs. Because of its many advantages over other materials for microfluidic devices, such as its ease of fabrication, solvent compatibility, transparency and availability, PDMS is chosen as the base material for our microfluidic device. Combined with the desirable optical properties of the NPLs, PDMS can provide easy integration of laser media into flexible microfluidic networks. Using capillary as well as pressure-driven flows, record low optical gain thresholds were achieved. Using capillary forces, single-mode lasing was demonstrated on an on-chip Fabry-Pérot cavity from red-emitting NPLs. The use of pressure-driven 
flow allowed for the observation of gain from a liquid-liquid waveguide. These microfabricated NPL solution lasers have the potential to provide compact and inexpensive coherent light sources for applications in microfluidics and integrated optics.

Keywords: colloidal semiconductor nanocrystals, microfluidics, microfluidic waveguides, optical gain, Fabry-Pérot laser. 


\title{
ÖZET
}

\section{KOLOIDAL YARIILETKEN KUANTUM KUYULARININ OPTIK MIKROAKISKAN DALGAKILAVUZU VE SIVI LAZERLERI}

\author{
Joudi Maskoun \\ Malzeme Bilim ve Nanoteknoloji, Yüksek Lisans \\ Tez Danışmanı: Hilmi Volkan Demir \\ İkinci Tez Danısmanı: Emine Yegân Erdem
}

July 2020

Mikroakışkanlar, bakım-noktası-sistemleri, çip üzerinde laboratuvar (LOC) cihazları ve ilaç dağıtımı ve ayrıştırılması gibi birçok uygulama sunan önemli bir teknoloji platformu haline gelmiştir. Bu teknolojinin tam potansiyeline ulaşması için ve uygulama yelpazesini genişletmek amacıyla birçok bileşen kullanılmakta ve iyileştirilmesi üzerine çalışılmaktadır. Mikroakışkanların önemli uygulamalarından birisi de mikroakışkan lazerlerdir. Mikroakışkan lazerler akış sitometrisinde sensor olarak ve kimyasal ve biyolojik reaksiyonları kontrol ederken ışık kaynağı olarak kullanılmaktadır. Literatürde, florosan boyaların kullanıldığı mikroakışkan sıvı-sıvı dalga kılavuzlarında lazer ışıması ve çoğaltılmış anlık ışıma bildirilmiştir. Bu cihazların performansı lazer için arzu edilen optik özelliklere sahip olan nano-levhalar olarak da bilinen kolloidal yariletken kuantum kuyuları kullanılarak önemli ölçüde geliştirilebilir. Bu tez çalışmasında, önceki çalişmalardan farklı olarak, polidimetilsiloksan (PDMS) ile kapsüllenmiş bir mikroakışkan kanalda Fabry-Pérot boşluğu kullanılarak koloidal nano-levhalar ile sıvıda-lazer elde edilmiş̧ir. Mikroakışkan cihazlar yumuşak litografi kullanılarak imal edilmiştir ve nano-levhalardan optik kazancın gözlemlenmesi için bir platform olarak kullanılmıştır. Üretim kolaylı̆̆ı, çözücü uyumluluğu, şeffaflık ve bulunabilirlik gibi birçok avantajı nedeniyle PDMS mikroakışkan cihazımız için temel malzeme olarak seçilmiştir. PDMS esnek yapısı sayesinde lazer ortamının mikroakışkan ağlara kolay entegrasyonunu sağlayabilir. Rekor seviyede düşük optik kazanç eşikleri kılcal ve basınçla çalışan akışlar kullanılarak elde edilmiştir. Tek modlu kırmızı lazer ışımaları kılcal kuvvetler kullanılarak çip üstü Fabry-Pérot çınlaç yapısında gösterilmiştir. Basınçla çalışan akış kullanılalarak, sıvı-sıvı dalga kılavuzundan optik kazanımın gözlenmiştir. Bu mikro-fabrikasyon 
yöntemiyle elde edilmiş nano-levha bazlı sıvı lazerler, mikroakışkanlar ve tümleşik optik uygulamaları için kompakt ve ucuz ışı kaynakları olmaya adaydır.

Anahtar sözcükler: kolloidal yariletken nanokristaller, mikroakışkanlar, mikroakışkan dalgakılavuzları, optik kazanç, Fabry-Pérot lazer. 


\section{Acknowledgement}

I would like to express my deepest appreciation to Prof. Hilmi Volkan Demir and to Prof. Emine Yegân Erdem for their guidance and support throughout my thesis work. I am also grateful for the members of Demir research group, and for Prof. Çağlar Elbüken for his helpful remarks.

The success and completion of my thesis would have not been possible without the continuous help and guidance of Dr. Negar Gheshlaghi, Malik Abdul Wahab, Dr. Savas Delikanli, Furkan Isik, and Dr. Onur Erdem. I would like to extend my sincere thanks to Melis Ozkan for all her support and the good times we spent together.

I am deeply indebted to my family, my mother Raha, my father Bader, and my siblings Bana, Sara, and Mohammed, for their unwavering care, unparalleled love, and for having faith in me. Without them, I would not be where I am today. I am extremely grateful to my sister Bana Maskoun for her continuous support and patience, and to Bouthaina Aoudi, who helped me through the hard times and been my family away from home over the years.

Finally, my very special thanks go to Haris Kahkeci for his unwavering encouragement and love, and for always believing in me and being by my side. I am forever grateful for his presence, love, and support. 


\section{Contents}

1 Introduction 1

1.1 Organization of the Thesis . . . . . . . . . . . . 3

2 Background 4

2.1 Photonics .......................... 4

2.1.1 Total internal reflection . . . . . . . . . . . . 4

2.1.2 Optical waveguiding ............... 6

2.1.3 Spontaneous and stimulated emission . . . . . . . . . 7

2.1.4 Lineshape function . . . . . . . . . . . . 8

2.1.5 Lasing ..................... . . . . 9

2.1.6 Fabry-Pérot cavity ................ 10

2.2 Colloidal Quantum Wells (CQWs) . . . . . . . . . . . 12

2.2.1 Quantum confinement in nanomaterials . . . . . . . 12

2.2.2 Heterostructures of CQWs ............ . 14 
2.2.3 Colloidal synthesis of CQWs . . . . . . . . . . . 16

2.3 Microfluidics . . . . . . . . . . . . . . . . . . . . . 18

2.3.1 Fluids . . . . . . . . . . . . . . . . . 18

2.3.2 Fluid flow in micro-channels . . . . . . . . . . . . . . . 22

$\begin{array}{lll}3 & \text { Experimental methods } & 27\end{array}$

3.1 Microfluidic device fabrication . . . . . . . . . . . . . . 27

3.1.1 Photolithography . . . . . . . . . . . . . . . 28

3.1.2 Soft lithography . . . . . . . . . . . . . . 31

3.2 Mirror deposition and characterization . . . . . . . . . . . . 32

3.2.1 Deposition techniques ............... 33

3.2.2 Mirror characterization . . . . . . . . . . . . 36

3.3 Microfluidic device operation . . . . . . . . . . . . . . . 38

3.3.1 Chemical compatibitily .............. 38

3.3.2 Plasma bonding and operation . . . . . . . . . . . 39

3.3.3 Contact angle measurements . . . . . . . . . . . . . . 40

3.4 Colloidal semiconductor nanocrystal characterization . . . . . . . 41

3.4.1 Absorption spectroscopy ................ . 41

3.4.2 Photoluminescence spectroscopy . . . . . . . . . . . 42

3.4.3 Electron microscopy . . . . . . . . . . . . . . . . . 43 
3.5 Optical gain measurements . . . . . . . . . . . . . . . 44

4 Optical gain and lasing in microfluidic devices 46

4.1 Motivation . . . . . . . . . . . . . . . . . 46

4.2 Sample preparation . . . . . . . . . . . . . . . . . . . 47

4.2 .1 CQWs synthesis . . . . . . . . . . . . . 47

4.2.2 Microfluidic device preparation and optimization . . . . . 50

4.3 Results and discussion . . . . . . . . . . . . . . . . . 51

4.3.1 CQW characterization .............. . . 51

4.3.2 Flow focusing and capillary flow . . . . . . . . . 53

4.3 .3 Mirror quality . . . . . . . . . . . . . 55

4.3.4 Optical gain measurements . . . . . . . . . . . . . 60

5 Conclusion and future outlook $\quad 64$

$\begin{array}{ll}\text { A Photolithography parameters } & 73\end{array}$ 


\section{List of Figures}

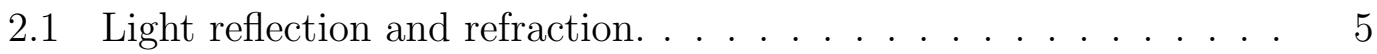

2.2 A waveguide traps an optical wave travelling through its core due to its higher index of refraction compared to the cladding. . . . . 6

2.3 Schematic of the microfluidic waveguide used in this study. . . . 7

2.4 Spontaneous emission (left), absorption (middle), and stimulated emission (right) phenomena. The arrow represents a photon with energy hv equal to the energy difference between energy levels $E_{2}$ and $E_{1} \ldots \ldots \ldots \ldots \ldots \ldots \ldots \ldots \ldots \ldots \ldots \ldots \ldots \ldots$

2.5 A Fabry-Pérot cavity of length d, a totally reflective mirror with a reflectance value $R_{1}$, and a partially reflective mirror with a reflectance value $R_{2} \ldots \ldots \ldots \ldots \ldots \ldots \ldots \ldots$

2.6 Demonstration of 0D, 1D, 2D, and 3D nanostructures. [16] . . 12

2.7 Energy bands in nanocrystals. . . . . . . . . . . . . . 13

2.8 Schematic of a CQW . . . . . . . . . . . . . . . . 14

2.9 CWQs heterostructures. (A) core, (B) core/shell, (C) core/crown, and (D) core/crown/shell CQWs. . . . . . . . . . . . . 15 
2.10 Photoluminescence (shaded) and absorption spectra of CdSe/CdS core/shell heterostructures with increasing shell thickness. [20] . . 15

2.11 La Mer Diagram. $[21] \ldots \ldots \ldots \ldots$

2.12 Contact angles $(\theta)$ and wettability. The surface on the left has a larger contact angle with the liquid, and therefore has a lower wettability, compared to the one on the right. The right surface is better wettable than the left one. . . . . . . . . . . . . 22

2.13 Fluid flow in (a) circular and (b) rectangular channels. The radius of the circular channel is $R$. The cross sectional area of the rectangular channel is $h \times w$. The length of both channels is $L$. . 23

2.14 Flow focusing. . . . . . . . . . . . . . .

3.1 Fabrication steps (a-c) of the microfluidic devices (d) . . . . 27

3.2 Photolithography. . . . . . . . . . . . . . . . . . 30

3.3 Soft lithography. . . . . . . . . . . . . . . . . . . . 31

3.4 Examples of wrinkled, cracked, and smooth thin gold films on PDMS. $[34] \ldots \ldots \ldots \ldots \ldots$

3.5 Schematic of the device orientation in the deposition chambers, where $\theta$ is the angle at which the device is tilted. . . . . . . 33

3.6 Thermal evaporation and sputtering using tilted sample configuration. . . . . . . . . . . . . . . . . . 34

3.7 Combinations of layered structures tested for Ag thin films for application as mirrors. . . . . . . . . . . . . . . 35

3.8 (a) Oxygen plasma activation of PDMS surface, and (b) bonding. 39 
3.9 Contact angle goniometer. . . . . . . . . . . . .

3.10 Absorption spectroscopy. . . . . . . . . . . . . . . . . . 42

3.11 Optical pumping and collection setup for gain measurements under one-photon absorption. . . . . . . . . . . . . .

4.1 Schematic illustration of our device fabrication steps. . . . . . . 51

4.2 (a) Photoluminescence (red) and absorption (blue) spectra, (b) TEM image, and (c) SEM image of 4 ML CdSe/CdS@Cd ${ }_{1-x} \mathrm{Zn}_{x} \mathrm{~S}$ core/crown@ 3 ML shell CQWs. (d) Photoluminescence (red) and absorption (blue) spectra, (e) TEM image, and (f) SEM image of 4 ML CdSe/CdS@CdZnS core/crown@ 4 ML shell CQWs. . . . . . 52

4.3 (a) Shows the ratio of the width of the focused stream to the total width of the channel as a function of the ratio of the volumetric flowrate of the focused stream to the total volumetric flowrate (b) Images of the focused streams at methylene blue volumetric flowrates of $5,10,15$, and $20 \mathrm{\mu L} / \mathrm{min}$, from left to right. . . . .

4.4 (a) Contact angle of toluene on treated PDMS, (b) fluorescent microscope image of capillary filled channel (shown with mirrors marked on the photograph) . . . . . . . . . . . .

4.5 \%oxidation over a period of $72 \mathrm{~h}$ for bare silver mirrors exposed and unexposed to oxygen plasma, and for $\mathrm{SiO}_{2}$ protected silver films exposed to oxygen plasma. . . . . . . . . . . . . . . . 57

4.6 (a-c) XPS spectra of silver mirrors exposed to oxygen plasma with oxidation protection layer. (d,e) XPS spectra of bare silver mirrors exposed to oxygen plasma. (a, d) Show Ag 3d spectra, (b,e) show the $\mathrm{O}$ 1s spectra, and (c) shows the Si 2p spectrum. . . . . . 58

4.7 SEM image of the deposited mirror. . . . . . . . . . . 59 
4.8 (a) Photoluminescence of $\mathrm{Cd}_{1-x} \mathrm{Zn}_{x} \mathrm{~S}$ core/crown@shell CQWs in capillary operation at increasing pump intensities. (b) Pump fluence $v s$ linewidth and output intensity. . . . . . . . . . . . . . 61

4.9 (a) Lasing of $\mathrm{Cd}_{1-x} \mathrm{Zn}_{x} \mathrm{~S}$ core/crown@shell CQWs in capillary. (b) Linewidth and luminescence of the output laser beam vs input pump intensity. (c) Image of the output laser beam. . . . . . . . . 62

4.10 (a) Photoluminescence spectra of 4 ML CdSe/CdS@ZnS core/crown@ 4ML shell CQWs in flow various pump intensities. (b) The change in luminescence and linewidth with increasing pump intensities. . 63 


\section{List of Tables}

2.1 Dimensionless numbers. $\quad \ldots \ldots \ldots \ldots$ 


\section{Chapter 1}

\section{Introduction}

The importance of microfluidics stems from its ability to control liquids at the microscale, as it is ideal for many systems including biological sensing and analysis which require higher precision and sensitivity. Microfluidic devices have been equipped to carry out several functions by integrating multiple microfluidic functionalities on single chips. The range of applications of these devices is constantly broadening with the continuous development of its components. One such advancement is the realization of microfluidic waveguides and lasers [1],[2],[3], [4].

Microfluidic gain media and lasers have been realized for various applications including reaction promoting, sensing, analysis, and light sources. Owing to the ability to easily design and fabricate numerous waveguide shapes and sizes, these devices provide the platform for exploring a rich variety of on-chip coherent light sources. Moreover, the laminar flow characteristic of microfluidics allows the flow of multiple streams with minimal mixing. Using laminar flow offers the opportunity of creating a rich variety of broadband light sources by flowing multicolor streams in the same waveguide.

This thesis introduces optical gain and lasing in microfluidic devices using colloidal semiconductor nanocrystals. The realization of a laser in a microfluidic device requires embedding an optical cavity to provide optical feedback and to 
confine emission. For this reason, a Fabry-Pérot (FP) cavity is created in the microfluidic device by depositing reflective mirrors on the channel walls.

Up until today, microfluidic lasers have been realized in FP and whispering gallery mode (WGM) cavities using fluorescent dyes as the gain materials [1],[2],[3],[4]. Whitesides et al. [1] report multimode lasing in an FP cavity from Rhodamine 640 at $1.1 \mathrm{~mJ} / \mathrm{cm}^{2}$. Moreover, an RGB laser with a threshold of 2.6 $\mathrm{mJ} / \mathrm{cm}^{2}$ has been demonstrated in a single FP cavity by flowing the fluorescent dyes Rhodamine 610, Coumarin 540, and Stilbene 420 side by side under laminar flow regime [4]. However, such fluorescent dyes photo-bleach or lose the ability to fluoresce due to irreversible photochemical reactions caused by photon generation [5] in short time scales [6], requiring continuous replenishment of the gain solution. This requirement demands complex operating conditions and excess material consumption as flow velocities used are as high as $100 \mu \mathrm{L} / \mathrm{min}$ to reduce the residence time of the gain material in the cavity [4].

We address these issues using colloidal semiconductor quantum wells (or CQWs) to enhance gain, reduce the threshold, and provide more photo-stable waveguides. CQWs are an important class of colloidal semiconductor nanocrystals (NCs), whose optical and electronic behaviors are excessively dependent on their dimensions, making it possible to tune their shapes, sizes, and compositions to realize the desired optical and electronic properties. As a result, these materials have found applications in light-emitting diodes (LEDs) [7], lasers [8], solar cells [9], biological labels[10], and many more.

However, colloidal semiconductor nanocrystals suffer from rapid Auger recombination, which limits their gain and thus their lasing performance. Auger recombination annihilates active carriers in a process of non-radiative exciton-hole recombination, where the exciton energy is transferred to other excitons in the form of kinetic energy [11]. This effect is suppressed in colloidal semiconductor quantum wells and thus they express superior optical properties as compared to other colloidal semiconductor nanocrystals.

In addition to the advantages of NCs, CQWs have a giant oscillator strength 
transition (GOST), which improves the linear and non-linear absorption crosssections and shortens the radiative decay time [12]. CQWs have also been proven to have giant modal optical gain capabilities [13] and no inhomogeneous broadening [14]. These many advantages make CQWs suitable for high-performance lasers and gain media.

In this thesis, optical gain of CQWs in solution is tested in a microfluidic platform. Moreover, a laser is realized CQWs by designing a microfluidic FabryPérot cavity which confines carrier emission and provides optical feedback through mirrors. The CQWs are introduced to the channel using two flow mechanisms, namely pressure-driven flow (PDF), which allows for the realization of multicolor, tunable waveguides and lasers, and capillary-driven flow (CDF).

CDF is shown for the first time in a microfluidic laser, and it has not been realized previously with fluorescent dyes, as they suffer from photo-bleaching. While CDF mechanism requires no external pumps and therefore has the advantage of ease of operation, it lacks the ability to tune the waveguide during operation, which is otherwise possible in PDF. The record low optical gain and lasing thresholds in solution observed in this work indicate that these devices provide a promising platform for exploring a rich variety of coherent broadband light sources.

\section{$1.1 \quad$ Organization of the Thesis}

We present a scientific background about waveguides and optical cavities, colloidal semiconductor quantum wells, and microfluidics, in Chapter 2 Sections 1, 2 , and 3 , respectively. The discussions include all the useful equations and physics behind the proposed work. In Chapter 3, we provide the experimental methods and device fabrication techniques followed for the realization of our devices. We present the results of our experiments in Chapter 4, and we finally conclude and review the future outlook of this work in Chapter 5. 


\section{Chapter 2}

\section{Background}

\subsection{Photonics}

Light is a form of electromagnetic (EM) waves, which are the propagation of energy, momentum, and angular momentum emitted by accelerating charged particles. EM waves have both electric and magnetic field components, which are described by Maxwell's equations. The electric and magnetic field components of light are in phase, and oscillate perpendicularly to each other and to the direction of the wave propagation. Light waves are governed by the wave function [15] shown below, which is a solution of Maxwell's equations.

$$
\nabla^{2} u-\frac{1}{c}=0
$$

\subsubsection{Total internal reflection}

When electromagnetic waves encounter a material, optical processes of absorption, reflection, transmission, refraction, or diffraction may take place. Scattering, which is a process that causes the beam to split into several beams with different directions, may also occur. In absorption, electrons absorb the incident optical energy and are excited to a higher energy state. Reflection refers to the wave 
bouncing off, and transmission refers to the wave passing through the medium. Refraction is the process of light bending in the medium, and diffraction is the process of bending around the edges of the medium.

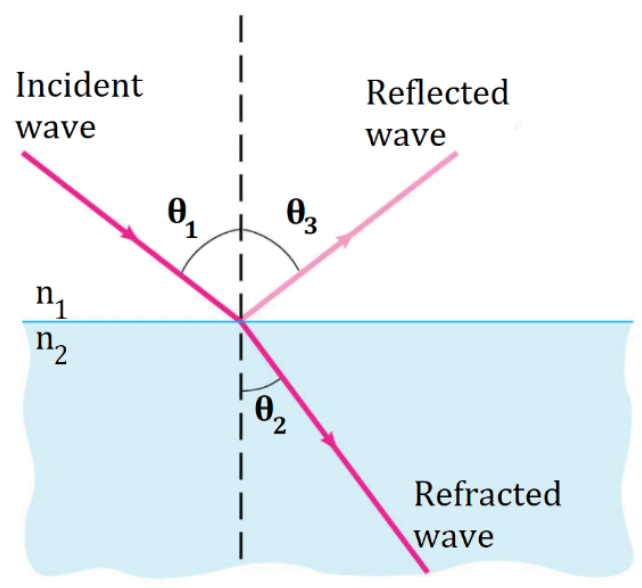

Figure 2.1: Light reflection and refraction.

When light traveling in medium 1 encounters another medium 2, a portion of the electromagnetic wave is refracted to medium 2 , and the remaining is reflected back into medium 1, as shown in Figure 2.1. Assuming that the light hits medium 2 at an angle $\theta_{1}$, the angle of reflectance, $\theta_{3}$, is equal to the angle of incidence, $\theta_{1}$, and the angle at which the light is refracted, $\theta_{2}$, may be calculated using Snell's law $[15]$ :

$$
n_{1} \sin \theta_{1}=n_{2} \sin \theta_{2}
$$

Internal reflection is achieved when $n_{1}>n_{2}$. The portion of the incident wave that is reflected back to medium 1 depends on the angle of incidence. If the angle of incidence is zero, the portion of reflected light, $r$, is calculated as follows [15]

$$
\frac{n_{1}-n_{2}}{n_{1}+n_{2}}=r
$$

As the angle of incident light increases, the reflection also increases gradually until it reaches unity at a critical angle $\theta_{c}$, at which a special case of reflection known as total internal reflection occurs, and all the incident light is reflected 
back to medium 1. The critical angle may be derived from Snell's law and has the form $[15]$ :

$$
\theta_{c}=\sin ^{-1}\left(\frac{n_{2}}{n_{1}}\right)
$$

When the angle of incidence is equal to or greater than the critical angle, total internal reflection occurs. The reflected light is accompanied by a phase shift that also depends on the incident and critical angles, and it varies from 0 to $\pi$ as the angle varies from 0 to $\theta_{c}$.

\subsubsection{Optical waveguiding}

To transfer light through long distances with little or no loss, the principle of total internal reflection is utilized in waveguides. Optical waveguiding works by trapping light in a medium of high index of refraction, sandwiched by another medium of lower index of refraction, as shown in Figure 2.2. In this structure, multiple total internal reflections of light occur and allow the wave to travel longer distances with minimal loss.

\begin{tabular}{|ll|}
\hline $\mathrm{n}_{1}$ & Cladding \\
\hline $\mathrm{n}_{2}>\mathrm{n}_{1}$ & Core \\
\hline $\mathrm{n}_{1}$ & Cladding \\
\hline
\end{tabular}

Figure 2.2: A waveguide traps an optical wave travelling through its core due to its higher index of refraction compared to the cladding.

Waveguides can take many shapes and forms, and may be designed to confine light in one or more dimensions, depending on the application. In this work, a rectangular microfluidic channel acts as a waveguide and allows light to propagate through one dimension, $\mathrm{z}$, while confining it in the other two, as shown in Figure 2.3 . 


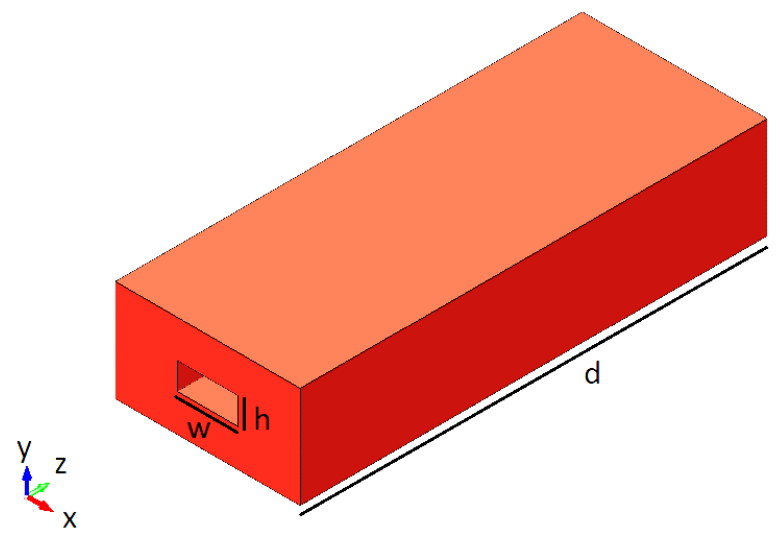

Figure 2.3: Schematic of the microfluidic waveguide used in this study.

\subsubsection{Spontaneous and stimulated emission}

The energy of electromagnetic modes is quantized, or can only take discrete rather than continuous values. A photon is defined as the quantum of electromagnetic radiation, or the smallest discrete amount of light. A photon with a frequency $\nu$ carries the amount of energy shown in Equation 2.5, where $h$ is Planck's constant and has the value $6.63 \times 10^{-34} \mathrm{~J} \cdot s$. A mode containing no photons has a zeropoint energy $E_{0}$ with a value of $1 / 2 h \nu$, consequently, a mode carrying n photons has the amount of energy shown in Equation 2.6 [15].

$$
\begin{gathered}
E=h v \\
E_{n}=\left(n+\frac{1}{2}\right) h v
\end{gathered}
$$

An atom with energy levels $E_{1}$ and $E_{2}, E_{2}$ being an excited energy state, can interact with and emit energy in the form of photons. Depending on the initial state of the atom, the photon-atom interaction may take the form of absorption, spontaneous emission, or stimulated emission, as shown in Figure 2.4.

Spontaneous emission, or "luminescence" may occur when an atom spontaneously releases its energy in the form of a photon and falls from an excited energy state to a lower energy state. The emitted photon will have an energy equal to the difference between $E_{2}$ and $E_{1}$. 


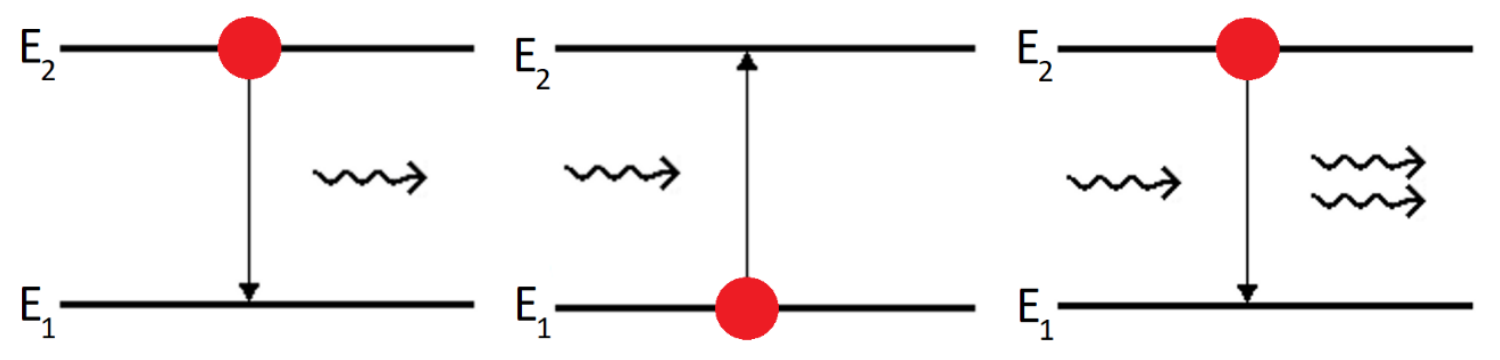

Figure 2.4: Spontaneous emission (left), absorption (middle), and stimulated emission (right) phenomena. The arrow represents a photon with energy hv equal to the energy difference between energy levels $E_{2}$ and $E_{1}$.

Absorption occurs when an atom at its ground energy state is excited to a higher energy state by photon absorption. This phenomenon occurs if an atom absorbs a photon with energy equal to the energy difference between its ground and excited energy state. The rate of absorption depends on the number of photons that are capable of causing the transition and the probability density is greater by $n$ times if $n$ photons are present in the mode [15].

The basic phenomenon of laser amplifiers is the spontaneous emission, and it occurs when an atom at an excited energy state encounters a photon which stimulates it to decay into the ground state and to release a photon identical to the incident photon. This occurs if the incident photon energy is equal to the energy difference between the ground and the excited state. When the number of photons that exist in the same mode increases, the rate of stimulated emission increases, and a laser is realized when stimulated emission is amplified in a cavity.

\subsubsection{Lineshape function}

The atom-photon interaction in a medium is defined by a spectral lineshape function, which is a function that describes the distribution of photon frequencies in the emission spectrum around the central frequency. Depending on the medium under study, the lineshape function may take different forms including Gaussian and Lorentzian functions. 
The most important characteristic feature of a spectral lineshape function is its transition linewidth, or full width at half maximum (FWHM), which describes the breadth of frequency distributions which arise from energy transitions. The FWHM is generally largest at the spontaneous emission regime, and significantly drops when optical gain is achieved.

Energy transitions contribute to the emission linewidth, and may occur by radiative means including the processes described earlier in Section 2.1.3, or by nonradiative means which cause homogeneous and inhomogeneous broadening. Homogenous broadening may result from inelastic collisions between atoms and container walls, or phase interruption, and inhomogeneous broadening arises from the different atoms that constitute a medium.

\subsubsection{Lasing}

Optical amplifiers are devices that amplify, or increase the amplitude of, input optical signals. These devices are usually used for intensifying weak signals of light travelling in optical fibers, and as parts of lasers. Each amplifier has a fixed amplifier gain, or a factor by which it increases the input signal. The LASER process, or light amplification by stimulated emission or radiation, takes advantage of a light amplifier, or active medium, placed in a cavity, or two parallel mirrors, and stimulated by a pump source.

Absorption, spontaneous emission, and stimulated emission occur simultaneously in matter. When a medium is in thermal equilibrium, the rate of absorption is larger than the rate of stimulated emission, and the light is attenuated. Laser amplification, however, occurs when the medium is in a non-equilibrium state, or when the rate of stimulated emission is larger than that of absorption.

By virtue of stimulated emission, a photon may produce a clone photon with the same frequency, direction, and polarization. These photons may then stimulate more emission, resulting in coherent light amplification. Since stimulated emission occurs with specific incident photon energies, the process is restricted 
to only certain frequencies.

In other words, the presence of more atoms at the excited energy state, or population inversion, must be achieved for laser amplification to occur. This process requires the use of an external source of power, or a pump, to induce stimulated emission. The pump may be electrical, chemical, or optical, and must provide enough energy to achieve population inversion.

As spontaneous emission would still exist in an amplifier, the signals from this emission account for noise. A laser amplifier may therefore be characterized by its gain, line width, phase shift, power source, gain saturation, and noise [15].

\subsubsection{Fabry-Pérot cavity}

An optical resonator is a device that confines and stores certain resonant frequencies of light. It is usually composed of a waveguide with two mirrors that allow light to be reflected with little or no loss to the surroundings. A simple one-dimensional resonator with parallel flat mirrors separated by a distance $d$ is shown in Figure 2.5, and is known as a Fabry-Pérot (FP) etalon. The components of an FP resonator include a gain medium where stimulated emission occurs, a pump to provide an incident photon source, and a cavity consisting of a partially reflective mirror, and a totally reflective mirror, to provide optical feedback.

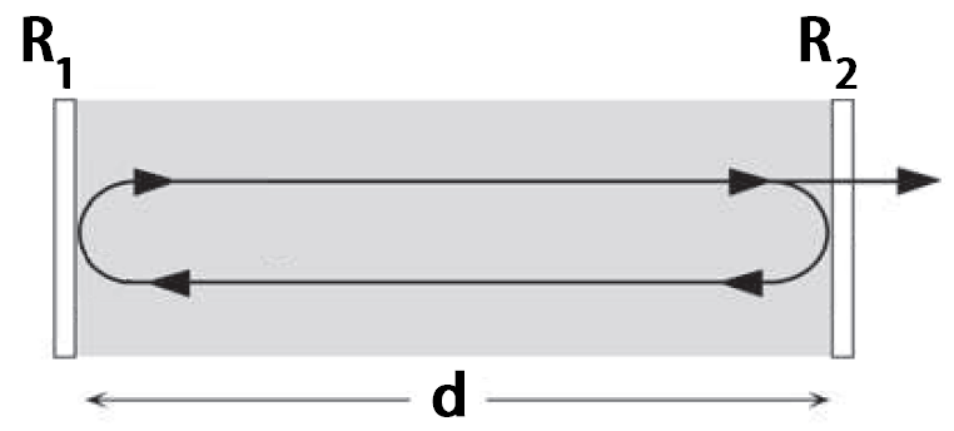

Figure 2.5: A Fabry-Pérot cavity of length d, a totally reflective mirror with a reflectance value $R_{1}$, and a partially reflective mirror with a reflectance value $R_{2}$. 
For lasing to take place in this resonator, the emissions need to build up until they reach a steady state, at which the optical power of the photons does not change after a full round trip in the cavity. The value of the gain coefficient that satisfies this condition at the steady state is called the threshold gain coefficient [15].

In order for lasing to occur, the gain and the phase conditions must be satisfied. The gain condition requires the gain coefficient to be greater than the loss coefficient, and the population difference must exceed a threshold value $N_{t}$. Losses from absorption and scattering exist in a laser amplifier, and these losses decrease with decreasing the cavity length, $\mathrm{d}$, and with increasing the reflectance values of the mirrors. 


\subsection{Colloidal Quantum Wells (CQWs)}

\subsubsection{Quantum confinement in nanomaterials}

Nanomaterials are a very important class of matter due to their outstanding properties, and it is important to understand the scientific background from which these properties emerge. Nanomaterials may be classified based on the nature of the carrier confinement into 0D, 1D, 2D, or 3D materials, as shown in Figure 2.6 [16]. OD nanomaterials confine electrons in all 3 dimensions, 1D nanomaterials confine electrons in 2 dimensions, and so on.

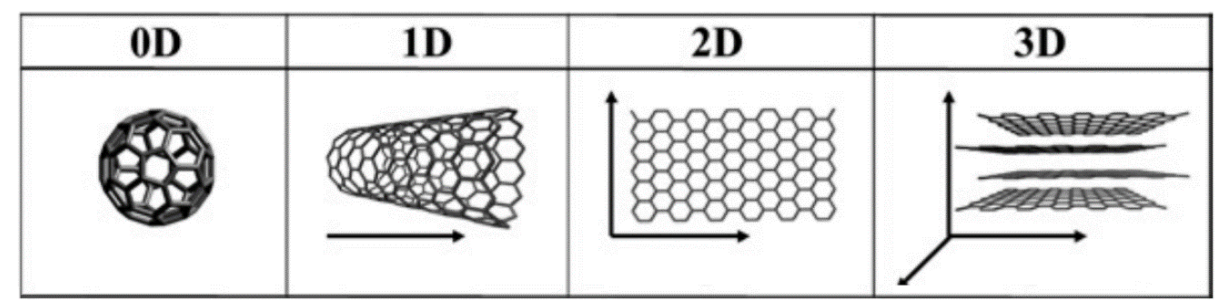

Figure 2.6: Demonstration of 0D, 1D, 2D, and 3D nanostructures. [16]

Electron confinement is crucial to the material properties, as it changes the band gap of the material. As the material size decreases, the energy levels become more discrete, and the band gap, $E_{g}$, increases, as shown in Figure 2.7. This confinement changes the properties of electrons, holes, and excitons in semiconductor nanomaterials, resulting in size and shape dependent properties. Such properties include absorption and emission spectra, recombination rates, and relaxation times [17].

To understand the effect of the spatial confinement, one must study the concepts of exciton formation and the Bohr radius. The Bohr radius may be calculated as shown in Equation 2.7, where $a_{0}$ is the hydrogen atom Bohr radius, $\epsilon$ is the medium dielectric constant, $m_{e}$ is the electron rest mass, and $m^{*}$ is the reduced mass of the electron hole pair [18].

$$
a_{B}=\epsilon a_{0} \frac{m_{e}}{m^{*}}
$$




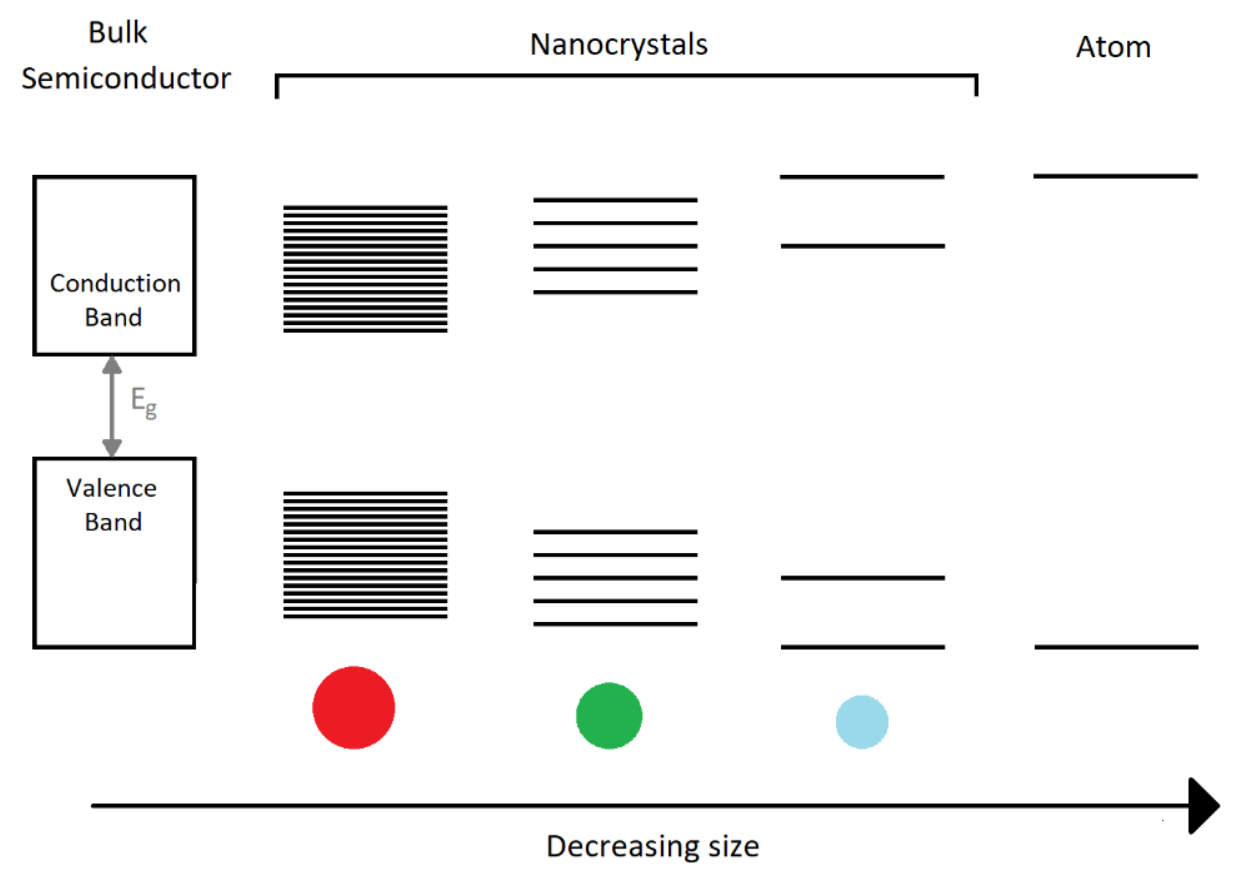

Figure 2.7: Energy bands in nanocrystals.

When an electron is excited to a higher energy level, it is kicked to the conduction band, leaving a hole behind. If the size and the exciton Bohr radius of the nanocrystal are comparable, quantum confinement is observed, and this electron and hole pair forms an "exciton" due to their Coulombic attraction. Quantum confinement increases the band gap in materials by restraining the motion of the carriers. Therefore, changing the size of the same material changes its band gap and hence its properties.

The band gap energy of a 0D semiconductor nanocrystal may be calculated as shown in Equation 2.8, where $E_{\text {confinement }}$ and $E_{\text {exciton }}$ are the confinement and exciton energies, and are defined in Equations 2.9 and 2.10, respectively [19]. It can also be confirmed from Equation 2.8 that reducing the size $(\mathrm{R})$ of the nanocrystal increases the energy gap, as explained earlier in this section.

$$
\begin{gathered}
E_{g}=E_{\text {bulkbandgap }}+E_{\text {confinement }}+E_{\text {exciton }} \\
E_{\text {confinement }}=\frac{\hbar^{2} \pi^{2}}{2 R^{2}}\left(\frac{1}{m_{e}}+\frac{1}{m_{h}}\right)
\end{gathered}
$$




$$
E_{\text {exciton }}=-\frac{1.8 e^{2}}{\epsilon R}
$$

\subsubsection{Heterostructures of CQWs}

Colloidal semiconductor quantum wells are 2D semiconductor nanomaterials, and are also referred to as nano-platelets because of their shape, shown in Figure 2.8. These materials have a nanometer scale vertical thickness, much lower than the exciton Bohr radius, and the quantum confinement is therefore achieved in only one dimension. The properties of the CQWs can be tuned by changing the spatial confinement, or controlling the number of monolayers. Increasing the number of monolayers increases the thickness of the CQWs, reduces the confinement, and therefore results in a red shift of the emission and absorption spectra.

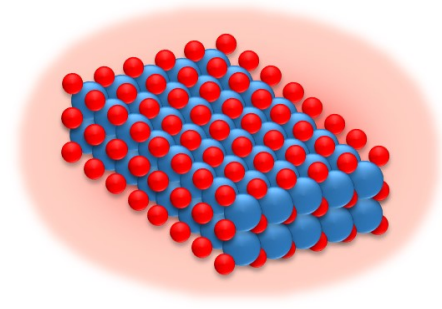

Figure 2.8: Schematic of a CQW.

The thickness of the CQWs is precisely controllable by minor alterations in the synthesis procedure. Consequently, the nanoparticles produced per batch are all of the same vertical thickness, known as "magic site", and have purely homogeneous broadening, accounting for their sharp photoluminescence (PL) peaks and narrow linewidths. In order to improve the quantum efficiency that arises from non-radiative decay and surface trap states in NCs, heterostructures of CQWs have been realized. Such hetero-structures include combinations of core, crown, and/or shell, as shown in Figure 2.9.

The growth of a crown around the core improves the PL, quantum yield, and stability of the resulting nanocrystal. Moreover, adding a shell sandwiches the 
a

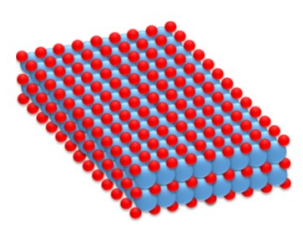

b

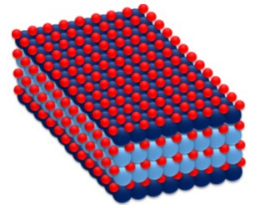

C

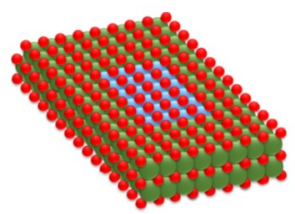

d

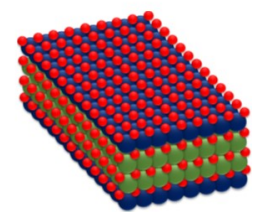

Figure 2.9: CWQs heterostructures. (A) core, (B) core/shell, (C) core/crown, and (D) core/crown/shell CQWs.

structure in the vertical direction, and contributes to the quantum confinement while improving the charge localization. The effect of adding shells to the CQW cores is shown in Figure 2.10 [20]. Furthermore, due to the numerous energy states available in CQWs, their absorption spectra are continuous for wavelengths shorter than their PL. Accordingly, CQWs are able to absorb photons of higher energies, as can be seen from the absorption spectra in Figure 2.10.

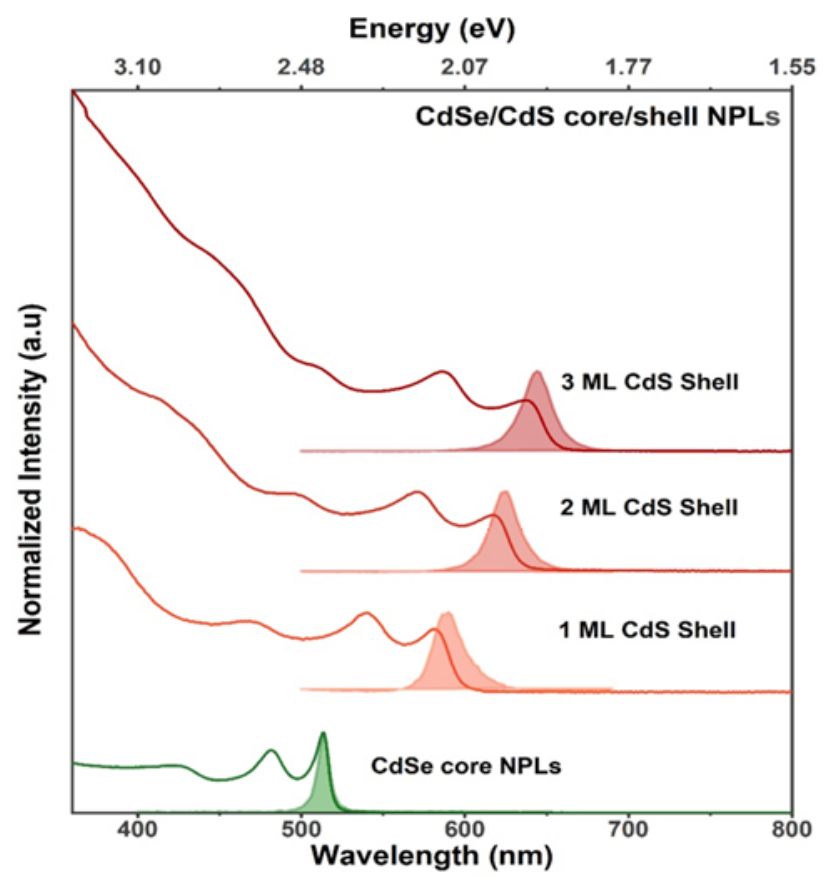

Figure 2.10: Photoluminescence (shaded) and absorption spectra of CdSe/CdS core/shell heterostructures with increasing shell thickness. [20]

By virtue of heterostructures, CQWs of Type I, Type II, and Quasi-Type II may be realized. Type I CQWs have electrons and holes confined in the core, and 
exhibit a red shift in the gain regime due to the attractive exciton interactions. On the other hand, Type II CQWs have the electrons and holes confined in different regions of the structure, resulting in a charge delocalization capable of suppressing Auger recombination, and repulsive exciton interactions. As a result, these materials exhibit a blue shift in the gain regime. Quasi-Type II CQWs have one confined carrier while the other carrier is free.

\subsubsection{Colloidal synthesis of CQWs}

The synthesis of CQWs must result in nanocrystals monodisperse in thickness, due to the strong dependence of nanocrystal properties on their size. In this thesis work, the CQWs used were prepared in a lab-scale batch process, using ligands, solvents, and precursors. The CQWs synthesis process occurs in 3 stages, as shown in the La Mer diagram shown in Figure 2.11 [21].

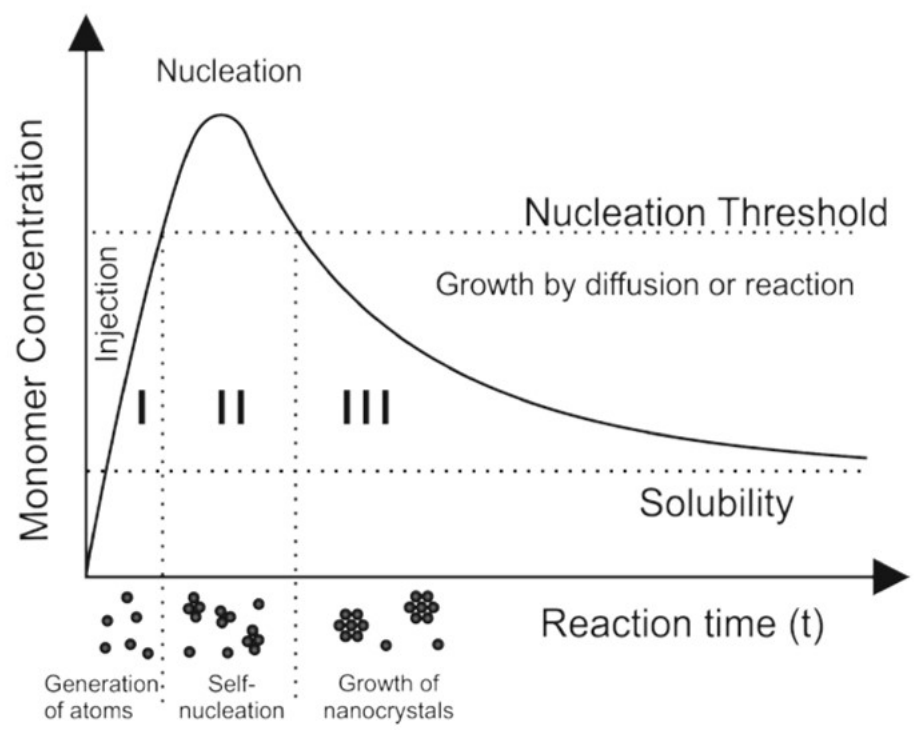

Figure 2.11: La Mer Diagram. [21]

The solvent is the medium where the reactions take place, and the ligands are used to control the stability and the final size of the NCs by preventing uncontrolled nanoparticle growth. Moreover, the ligands control the solubility 
and reactivity of the NCs, allow them to be suspended in solution and provide surface passivation [22].

In the first stage, precursors are injected into the system, and are decomposed into monomers, which act as a feed stock for nanocrystal formation by nucleation. When the monomer concentration reaches the nucleation threshold concentration, nucleation stage starts.

In the third stage, the nucleation stops and the growth of the nanocrystals occurs by consuming the monomers in the solution. The growth stage ends when the concentration of the NCs reach the solubility limit. These stages are carefully controlled by temperature, as nucleation occurs only at elevated temperatures $[22]$. 


\subsection{Microfluidics}

\subsubsection{Fluids}

The term fluid is used to describe matter that deforms, or flows, under the application of a tangential force, or shear stress $(\tau)$. Depending on the behavior of the fluid upon the exertion of shear stress, the fluid can be classified as Newtonian or non-Newtonian. Newtonian fluids deform linearly with stress, as shown in Newton's Law of Viscosity in Equation 2.11. The rate of fluid deformation, or shear rate $(\gamma)$, is inversely proportional to the fluid's resistance to flow, or viscosity $(\mu)$. Non-Newtonian fluids exhibit non-linear stress-strain relationship, and their viscosities are usually defined by a power law [23].

$$
\tau=\mu \frac{d v}{d y}=\mu \gamma
$$

Some of the conditions assumed while solving for fluid flow in a channel include incompressibility and no slip at the boundaries. The no slip boundary condition states that at a solid-fluid interface, the velocity of the fluid at the solid boundary is always zero relative to the boundary, if the characteristic length of the channel is larger than $300 \mathrm{~nm}$ [24]. Liquids are usually assumed to have stable densities

during deformation, since their average intermolecular distances are usually small [25]. Therefore, liquids are assumed to be "incompressible".

\subsubsection{Governing equations}

To fully understand the factors affecting flow in a microfluidic channel, one needs to understand the basics of fluid mechanics. For incompressible, Newtonian fluids, the governing equations for flow are the Navier-Stoke's (NS) and the continuity equations, which may also be regarded as conservation of momentum and conservation of mass equations, are shown in Equations 2.12 and 2.13, respectively.

$$
\rho\left(\frac{\partial u}{\partial t}+u \cdot \nabla u\right)=-\nabla P+\rho \vec{\gamma}+\mu \nabla^{2} u
$$




$$
\frac{\partial \rho}{\partial t}+\nabla \cdot \rho u=0
$$

The left side of the Navier-Stoke's equation shows the local acceleration due to

the change of velocity $\left(\frac{\partial u}{\partial t}\right)$, and the convective acceleration due to the changes in velocity field $(u \cdot \nabla u)$. The right side of the equation shows the pressure gradient, external forces, such as gravity or surface tension, and viscous forces, respectively from left to right [23]. The equation of continuity states that the rates of the mass entering and exiting the system must be equal, where $\rho$ is the density of the fluid, and $u$ is its velocity.

\subsubsection{Dimensionless numbers}

The forces acting on a flowing fluid may be categorized into volume (or body) and surface, or contact, forces. Volume forces include gravitational, centrifugal, and magnetic, etc., and surface forces include viscous, pressure, and surface tension, etc.. The main forces that affect a fluid in motion are surface tension, inertial forces, gravitational forces, and viscous forces. The relative importance of these forces can be determined by dimensionless numbers, which help identify the flow regime. Some dimensionless numbers are shown in Table 2.1 [26], [27].

Table 2.1: Dimensionless numbers.

\begin{tabular}{l|l|c} 
Dimensionless number & \multicolumn{1}{c}{ Compares } & Equation \\
\hline Reynold's number, Re & Inertial to viscous forces & $R e=\frac{\rho u L_{c h}}{\mu}$ \\
Capillary number, Ca & Viscous forces to surface tension & $C a=\frac{\mu u}{\gamma}$
\end{tabular}

The forces that arise due to the frictional shear forces present between the flowing layers in a fluid, or viscous forces, allow the fluid layers to flow each at a different velocity, producing a velocity gradient in the direction perpendicular to the flow. Viscous forces, when dominant, slow the fluid down and force it to flow in an orderly manner, where, at steady state, each layer of the fluid flows at a constant velocity. Inertial forces, on the other hand, work on keeping the 
fluid in motion. The ratio of the inertial force to the viscous force gives rise to a dimensionless quantity, called Reynold's number (Re), shown in Table 2.1.

Reynold's number identifies whether the flow is laminar or turbulent. Laminar flow is identified by the parallel layers of different velocities which slide past one another in a channel, and occurs as an effect of viscous forces. As viscous forces become negligible, and Reynold's number exceeds a certain value, inertial forces become dominant and the fluid flow becomes turbulent. Turbulent flows are identified by vortices and unsteady flow velocities.

The transition from laminar to turbulent flow occurs at a Reynold's number of 1500 [23]. As the dimensions of microfluidic channels are on the orders of micrometers, Reynold's number rarely exceeds the value of 100, and the flow is always in the laminar regime, meaning that inertial forces are negligible. Negligible inertial forces indicate the absence of acceleration, and that a constant force needs to be exerted for flow in a microchannel to occur.

Due to the low Reynold's number in microfluidic channels, the resulting flow is laminar, and mixing along the fluid layers is therefore only achievable by diffusion between the fluid layers. The driving force for diffusion is the presence of a concentration gradient, across which molecules move from regions of higher concentrations to those of lower concentrations.

Diffusion at steady state is quantitatively represented by Fick's first law of diffusion, which is shown for one-dimensional case in Equation 2.14, which defines the flux, $J$, as a function of the diffusion coefficient, $D$, and the concentration gradient along the $\mathrm{x}$ direction, $\frac{d c}{d x}$. The diffusion coefficient is a constant defined by the Stoke's Einstein law, where $k_{B}$ is the Boltzmann constant, $T$ is the temperature, $R$ is the particle radius, and $\mu$ is the fluid viscosity. The diffusion coefficient increases with temperature, as can be interpreted from Equation 2.15 [23].

$$
\begin{aligned}
& J=-D \frac{d c}{d x} \\
& D=\frac{k_{B} T}{6 \pi \mu R}
\end{aligned}
$$


At the microscale, since the surface to volume ratio is relatively large, the dominant forces that govern the fluid flow change. Capillary forces, which are usually neglected at the macroscales become important at the microscale, as they may sometimes be large enough to induce flow. To understand capillary forces, some basic understanding of surface effects and Gibbs free energy is required. Gibbs free energy is the thermodynamic potential energy of a system. As systems always act to reduce their energies, the equilibrium is always reached when the Gibbs free energy is at minimum. The molecules of any object have more unsatisfied bonds at the surface than in the bulk, and thus a higher Gibbs free energy.

Surface molecules therefore work to minimize their energies. Surface tension is a force that exists at the interface of a fluid with air, and acts to minimize the interfacial area to reduce the surface energy. Similarly, interfacial tension exists between a fluid-fluid or a fluid-solid interface, and sometimes may work on drawing the surface molecules into the bulk, to minimize the contact area at the interface. The interfacial tension is expressed by Wibowo et al. [25] as the Gibbs free energy per unit area, and has the units $\mathrm{J} / \mathrm{m}^{2}$ or $\mathrm{Pa} \cdot \mathrm{m}$.

At the microscale, these forces can produce surface deformations at fluid-fluid interfaces and cause droplet formations. Moreover, in a channel, when the surface tension between the fluid and gas interface is greater than the interfacial tension between the fluid and the solid, capillary flow occurs. The dominance of surface forces over viscous forces may be evaluated by the capillary number, Ca, shown in Table 2.1. For the surface tension between the fluid and the channel to be lower than that between the fluid and gas, the channel affinity to the fluid must be high.

The affinity may be measured as a function of channel wettability; as hydrophobic solvents may wet a hydrophobic channel. The wettability is related to how a droplet rests on a solid surface. Based on the surface energies, a droplet that highly wets the surface, would have a positive contact angle smaller than $90^{\circ}$, and one that partially wets the surface would have a contact angle between $90^{\circ}$ and $180^{\circ}$, as shown in Figure 2.12. 

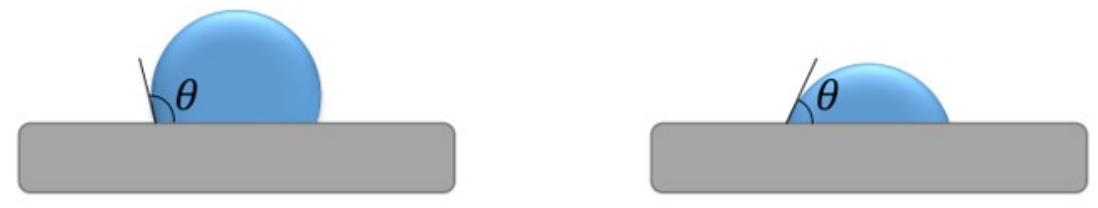

Figure 2.12: Contact angles $(\theta)$ and wettability. The surface on the left has a larger contact angle with the liquid, and therefore has a lower wettability, compared to the one on the right. The right surface is better wettable than the left one.

The contact angle is therefore a function of the surface energies of the liquid, solid, and gas in the system under study. It is also defined as shown below in Young's Equation [27], where $\gamma_{S G}, \gamma_{S L}$, and $\gamma_{L G}$ are the surface tensions between solid and gas, solid and liquid, and liquid and gas, respectively.

$$
\cos \theta_{c}=\frac{\gamma_{S G}-\gamma_{S L}}{\gamma_{L G}}
$$

\subsubsection{Fluid flow in micro-channels}

Flow in micro-channels is achieved mainly by three methods: namely, the application of a pressure gradient, capillary forces, and elecrtokinetic forces. This study applies capillary and pressure driven flows, and the theory is explained in this section by deriving solutions of the Navier-Stokes equation for our channels.

\subsubsection{Pressure driven flow}

Pressure driven flow, or PDF, refers to the flow that is initiated by creating a pressure gradient in the channel. Fluids flow from regions of high pressure to regions at low pressure, where pressure is usually applied using pumps. To develop an understanding of PDF, the Navier-Stoke's equation will be solved in this section considering flow in a circular microchannel, shown in Figure 2.13a, with a radius $R$ and a pressure gradient along the $z$ axis, and will then be extended to cover rectangular micro-channels. 

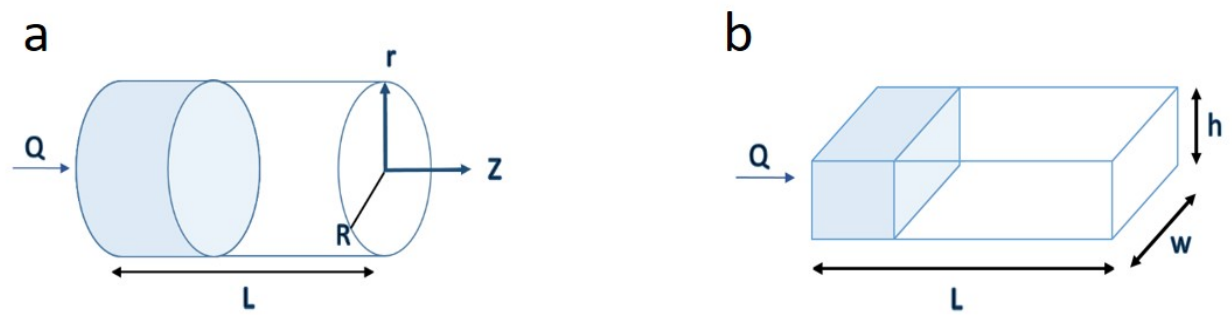

Figure 2.13: Fluid flow in (a) circular and (b) rectangular channels. The radius of the circular channel is $R$. The cross sectional area of the rectangular channel is $h \times w$. The length of both channels is $L$.

Since the pressure gradient is applied in one direction only, and since the flow is laminar, the velocity is only expected to be nonzero along the direction of flow. Therefore, the convective acceleration term also drops from the NS equation. Solving for the steady state laminar flow condition allows dropping the acceleration due to the term of velocity changes. The resulting simplified NS equation is obtained in Equation 2.17

$$
\nabla P=\mu \nabla^{2} u
$$

where, in cylindrical coordinates,

$$
\nabla^{2} u=\frac{\partial^{2} u}{\partial r^{2}}+\frac{1}{r} \frac{\partial u}{\partial r}+\frac{1}{r^{2}} \frac{\partial^{2} u}{\partial \theta^{2}}+\frac{\partial^{2} u}{\partial z^{2}}
$$

since the velocity only changes with varying $r, \nabla^{2} u$ further simplifies to:

$$
\nabla^{2} u=\frac{\partial^{2} u}{\partial r^{2}}+\frac{1}{r} \frac{\partial u}{\partial r}
$$

To solve this second order differential equation, two boundary conditions imposing no slip at the boundary $(u=0$ at $r=R)$, and the maximum velocity at the center $\left(\frac{d u}{d r}=0\right.$ at $\left.r=0\right)$ are applied to arrive at the velocity profile given in Equation 2.20 .

$$
u=\frac{1}{4 \mu} \frac{\Delta P}{L}\left(R^{2}-r^{2}\right)
$$

To find the volumetric flow rate, the velocity profile is integrated along the channel volume. The resulting equation is the Hagen-Poiseulle equation, where $R_{H}$ is the 
hydrodynamic resistance to flow.

$$
Q=\int d V u=\frac{\pi R^{4}}{8 \mu L} \Delta P=\frac{\Delta P}{R_{H}}
$$

The characteristic length in microfluidic channels is defined by the hydraulic radius shown below. This radius is used as an approximation for non-circular channels.

$$
R_{h}=\frac{2 A}{P}=\frac{w h}{w+h}
$$

For a rectangular channel, the hydrodynamic resistance, $R_{H}$, is approximated as [27]:

$$
R_{H}=\frac{12 \mu L}{w h^{3}}\left[1-0.63 \frac{h}{w}\right]^{-1}
$$

\subsubsection{Flow focusing}

In the case of pressure driven flow, as the major aim is to obtain a tunable soft wall around our gain medium, the effect of the flow rates on the soft wall width is analyzed. Since the gain medium is to flow in the center of the channel, it is said to be "focused", and the cladding fluid flows from the sides.

By changing the flow rate ratios, we can adjust the width of the channel occupied by our gain medium. Lee et al. [28] reported a model proposed for flow focusing in a cross junction microfluidic device. The ratio of the width of the focused stream, $w_{f}$, to the total width of the channel, $w_{o}$, will be equal to the volumetric flow rate of the focused stream, $Q_{f}$, to the total volumetric flow rate, as shown in Equation 2.24 and Figure 2.14. We assume that the streams do not mix due to the laminar flow condition and the short channels.

$$
\frac{w_{f}}{w_{o}}=\frac{Q_{f}}{Q_{o}}
$$




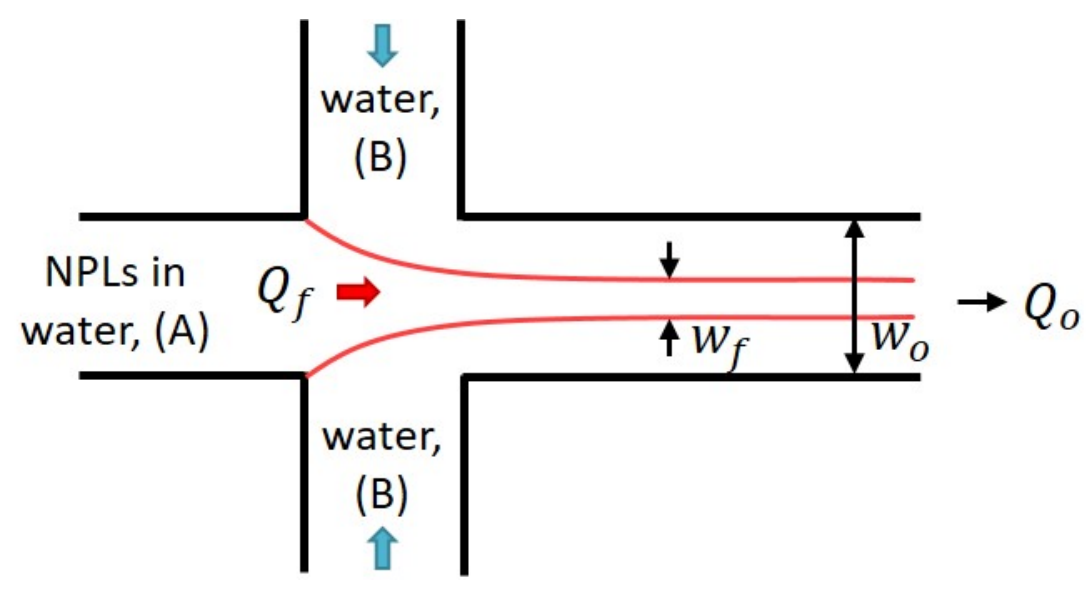

Figure 2.14: Flow focusing.

\subsubsection{Mixing by diffusion}

In this section, the mixing in a microfluidic channel with length $l$, width $w$, and height $h$, will be investigated. Fluid $\mathrm{A}$ is injected into the center of the channel, and is sandwiched by fluid B, as shown in Figure 2.14, creating concentration gradients along the $\mathrm{x}$ and $\mathrm{y}$ directions. The time required for species in fluid A to achieve complete mixing by diffusion is expressed in Equation 2.25, where $w_{f}$ is the width of the focused stream and $D$ is the diffusion coefficient. This equation implies that as the residence time of the fluids in the channel increases, the mixing increases [29].

$$
t_{m i x}=\frac{w_{f}^{2}}{\pi^{2} D}
$$

In this study, it is safe to assume that if the residence time of the fluid in the channel is less than the time required for mixing by an order of magnitude, then no mixing will occur.

\subsubsection{Capillary driven flow}

As in the analysis of pressure driven flow, the Hagen-Poiseuille equation for volumetric flow rate holds in the capillary driven flow. The pressure required for this analysis, however, is expressed by the Young-Laplace equation shown below, 
where $\theta_{t}, \theta_{b}, \theta_{l}$, and $\theta_{r}$ are the contact angles at the top, bottom, left, and right channel walls, respectively, and $h$ and $w$ are the channel height and width, respectively, and $R_{h}$ is the hydraulic radius. Simplifying Equation 2.26 [30], the pressure drop in a rectangular channel made of one material can thus be expressed by Equation 2.27 [27].

$$
\begin{gathered}
P=-\gamma\left(\frac{\cos \theta_{t}+\cos \theta_{b}}{h}+\frac{\cos \theta_{r}+\cos \theta_{l}}{w}\right) \\
\Delta P=\frac{2 \gamma}{R_{h}} \cos \theta
\end{gathered}
$$




\section{Chapter 3}

\section{Experimental methods}

\subsection{Microfluidic device fabrication}

The microfluidic devices used in this study were made of PDMS to provide easy integration of our device to microfluidic networks. PDMS is the most widely used material for many applications, owing to its transparency, inexpensiveness, and ease of sealing to glass or another PDMS layer. The devices were prepared by soft lithography technique, where patterns from a mold are transferred onto PDMS elastomer. The mold was prepared by the standard photolithography technique using negative photoresist and a dark field mask. Figure 3.1 shows an overview of the fabrication steps.
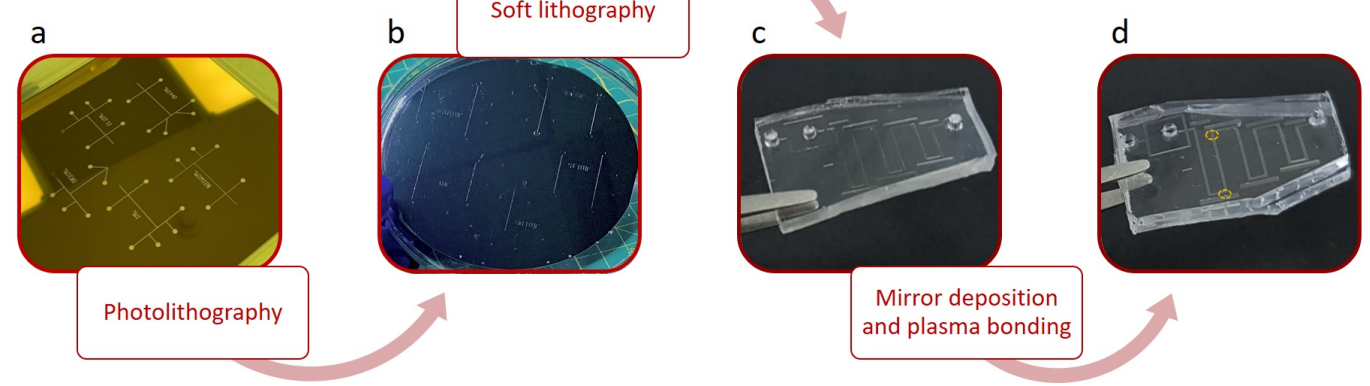

Figure 3.1: Fabrication steps (a-c) of the microfluidic devices (d). 


\subsubsection{Photolithography}

The desired features were drawn on Tanner L-edit and were transferred to a $5 \times 5$ inch glass mask. The mask prepared for this application was a dark field mask, which has transparent features, and is covered by chrome on the remaining parts, as shown in the Figure 3.1a. The chrome acts as a UV light barrier, and therefore the mask only allows light to pass through the drawn features. The pattern from the glass mask was then transferred onto a 4-inch silicon wafer.

After transferring the desired patterns to a dark field mask, photolithography was used to transfer the patterns from the mask to a silicon wafer. The term photolithography is derived from the words "photo", "litho", and "graphy", which are Latin for "light-stone-writing", or writing by light. Photolithography is achieved by making use of a light sensitive material, or photoresist (PR), and UV light for pattern transfer.

A conventional photolithography technique consists of cleaning a silicon wafer, spin-coating the PR on the wafer, baking the PR prior to exposing it to the mask, UV exposure using a mask aligner for pattern transfer, baking the PR after the UV exposure, and washing with developer to remove the undesired PR. This process is carried out in a cleanroom environment in order to prevent contamination to the patterns.

Cleaning the silicon wafer is important to remove any residual dust or contamination. Wafers contaminated with hard particles must be cleaned to prevent the formation of streaks, or non-uniform coating during the spin-coating of the photoresist. Following cleaning, the wafer should be dried to remove any moisture, which negatively affects the PR adhesion onto the wafer.

To obtain the desired PR thickness, the spin coater rotation speed and acceleration are adjusted according to the PR data sheet provided by the vendor for each PR. The thickness at a given acceleration and velocity is usually larger for PRs with greater solid percentage. After spin-coating the photoresist on a circular silicon wafer, an "edge bead" is usually observed, which is a visible increased 
thickness of photoresist at the edges of the wafer, and can be removed by acetone.

A photoresist is an organic polymer consisting of a base resin, a photoactive compound that responds to light, and a solvent which determines the viscosity. After baking and spin-coating, it may be assumed that the remaining photoresist on the wafer contains only solid contents. Photoresists are classified into positive or negative photoresists, based on the photoactive compound they contain [31].

Positive PRs contain photosensitive dissolution inhibitor, which, upon UV exposure, allows the PR to be dissolved. Negative PRs, on the other hand, contain photosensitive curing agents, which allow the polymer to crosslink and harden upon exposure. Therefore, upon the exposure of the PR by UV light, the exposed area stays after developing if the PR is negative, and is removed if the $\mathrm{PR}$ is positive [32]. In this work, a negative photoresist is used.

After spin-coating, the photoresist must be baked to evaporate the solvent and increase the PR density. To achieve a uniform evaporation, a hot plate is generally used, where the evaporation occurs from the bottom of the PR layer. Insufficient pre-exposure baking may result in weak adhesion of PR onto the wafer. The PR is then exposed to UV light for pattern transfer using a mask aligner. For our purpose, contact alignment is used for increased resolution.

Finally, the patterned photoresist is baked after UV exposure in order to stabilize and harden the PR. Longer post-exposure baking times makes the PR removal from the wafer harder, on the expense of reducing the sharpness of the PR edges. During the post-exposure baking step, the PR is heated to a temperature above its glass transition temperature, causing it to plastically reflow, therefore softening its sidewall angles [33].

A summary of the standard photolithography process using a negative PR is shown in Figure 3.2. The detailed procedure followed for our devices is explained in the remaining of this section, and the parameters used are shown in the Appendix. 


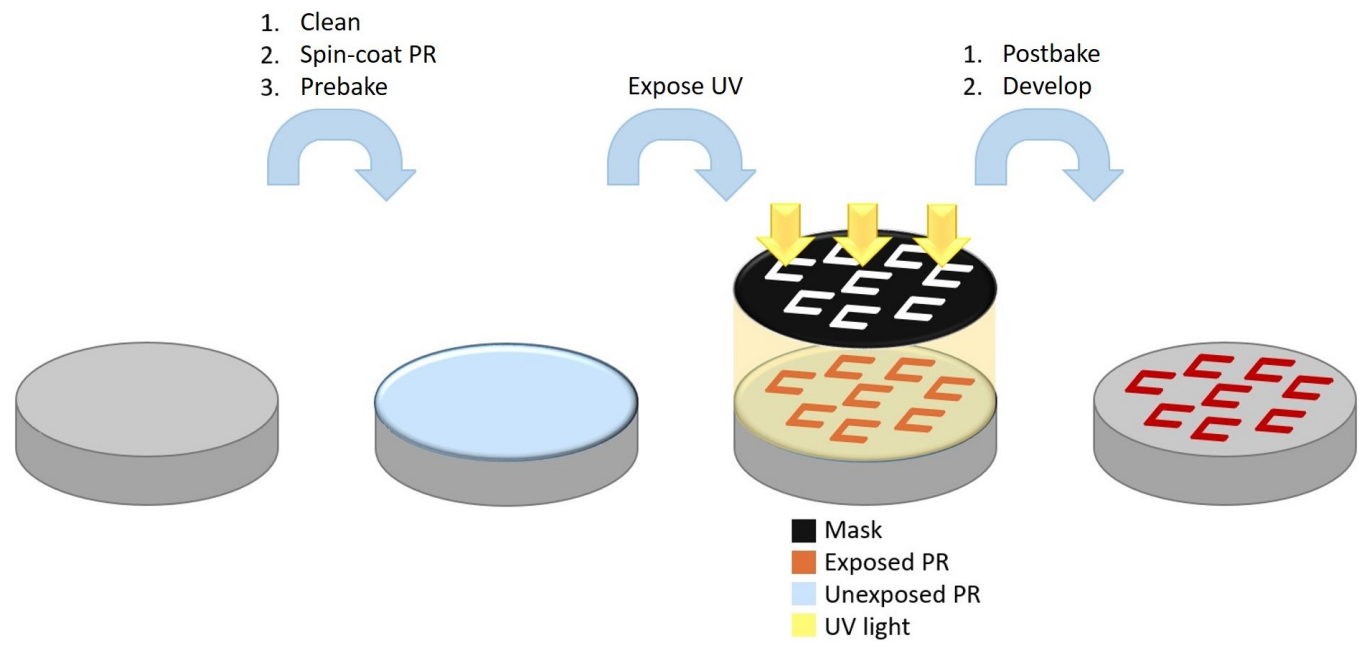

Figure 3.2: Photolithography.

In this work, two layers of photoresist were used to enhance the adhesion of the photoresist on the wafer, and therefore allow it to be used multiple times. SU8 2005 and SU8 2050 from Microchem were used as the photoresists for the base and main layers, respectively. The base layer acts as an adhesive layer, and the main layer contains the pattern. The parameters given in this section correspond to base layer and main layer PR film thicknesses of 2 and $100 \mu \mathrm{m}$, respectively.

First, a clean silicon wafer was obtained and its polished side was rinsed sequentially with acetone, IPA, and DI water, and then dried with $\mathrm{N}_{2}$ gas. To ensure that the moisture in the wafer was removed, the wafer was placed in an oven at $120{ }^{\circ} \mathrm{C}$ for $10 \mathrm{~min}$. The wafer was then allowed to cool to room temperature before starting the procedure.

For the base layer, SU8 2005 was spin-coated onto the wafer, then the wafer was transferred to a hot plate for a "pre-exposure bake". Finally, the base layer was exposed to a clear glass mask in a mask aligner. Since the SU8 2005 is a negative photoresist, it hardened after UV exposure. After exposure, the base layer was baked, and left to cool to room temperature.

The main layer that carries the microfluidic devices was prepared using SU8 2050. The same photolithography process was applied to this layer, with different spin-coater, pre-bake, mask aligner, and post-bake parameters. This layer was 
exposed to the mask that carried the desired features. The post-exposure bake of the main layer was followed by the development of the unexposed photoresist using SU8 2050 developer. The wafer was placed in a beaker which contains a sufficient amount of SU8 2050 developer to immerse the wafer and was slowly agitated for $10 \mathrm{~min}$.

The wafer was finally removed from the beaker and rinsed with IPA and water and dried with $\mathrm{N}_{2}$. If residual undeveloped SU8 was still observed, the development step was repeated with fresh developer for $30 \mathrm{~s}$. After the completion of photolithography, a silicon mold containing the desired features was obtained as in Figure 3.1b. From this mold, PDMS microfluidic devices were produced using soft lithography.

\subsubsection{Soft lithography}

Soft lithography is the process of transferring a pattern from one substrate to another. After the silicon master mold with the desired features was prepared by the means described earlier, PDMS was mixed with its curing agent at a ratio of 10:1 and was poured onto the mold. The mold was degassed in vacuum for 20 min to remove the air bubbles trapped in the PDMS elastomer and was then transferred to an oven at $80{ }^{\circ} \mathrm{C}$ and kept for $40 \mathrm{~min}$. The PDMS was then peeled off, and the microfluidic devices were cut out and their holes were punched. Then, mirrors were deposited if desired, and the surfaces of a PDMS device and a flat PDMS substrate were activated by oxygen plasma, and finally pressed together for adhesion. A summary of the process is shown in Figure 3.3.

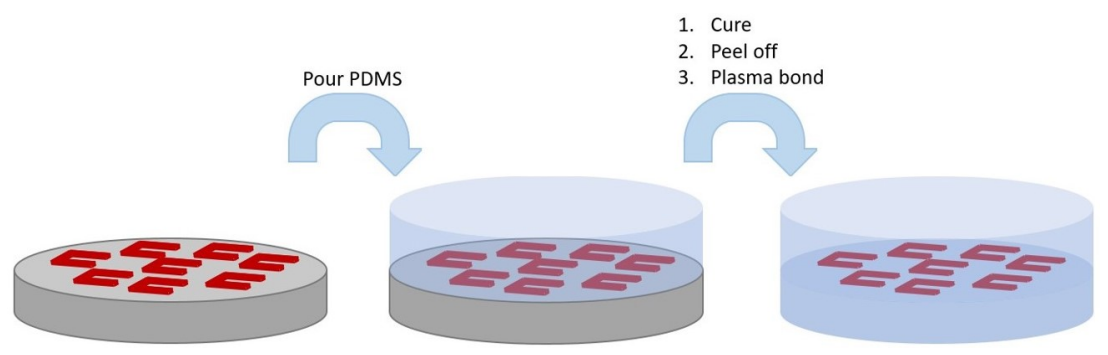

Figure 3.3: Soft lithography. 


\subsection{Mirror deposition and characterization}

A Fabry-Pérot cavity was created by depositing mirrors on the walls of the microfluidic device in a thermal evaporation chamber. The devices were mounted at $75^{\circ}$ angles, and the parts of devices where mirror deposition was undesired were carefully covered with thermal tape prior to deposition. The mirror deposition on the channel walls was achieved by tilting the microfluidic devices in the deposition chamber.

The deposition of an adhesion layer of titanium or chromium is required to obtain smooth mirror films on PDMS. Depending on the adhesion layer thickness, the deposited mirror films may be smooth, buckled, or micro-cracked, as shown in Figure 3.4 [34], and as our films were used as mirrors, the smooth morphology was desired for our application. To achieve this requirement, a study by Graudejus et al. [35] suggests that the thickness of the adhesion layer must be between 90 and $110 \AA$.
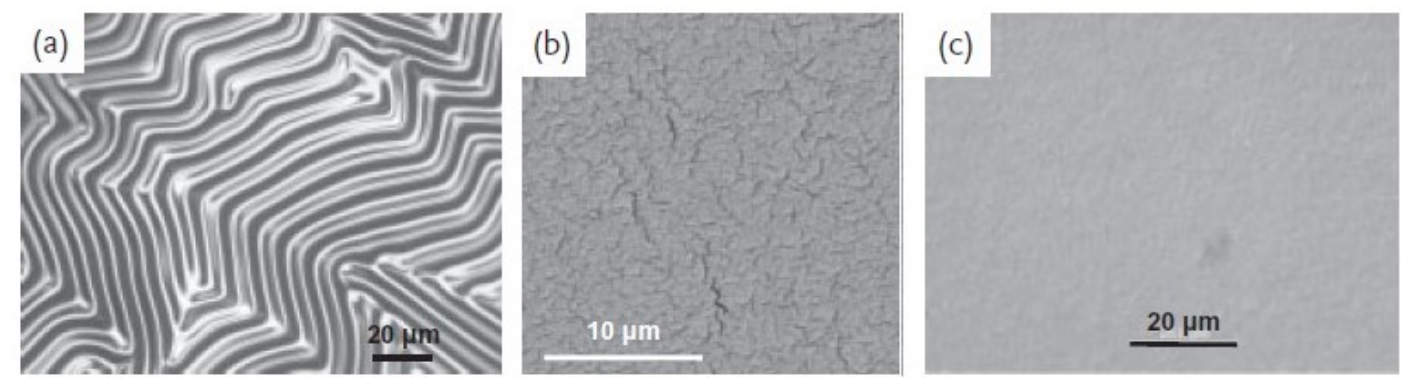

Figure 3.4: Examples of wrinkled, cracked, and smooth thin gold films on PDMS. $[34]$

As only the walls of the channels were to be covered, each side of the channel was coated at a time. The channels were mounted on angled sample holders made of PDMS, as shown in Figure 3.5. 


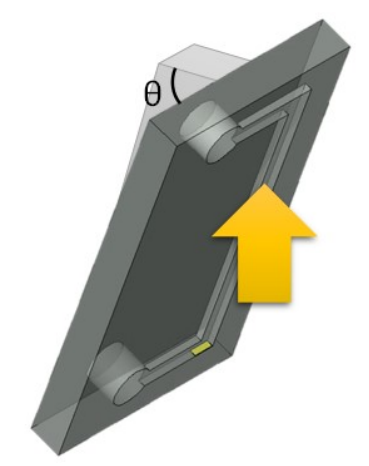

Figure 3.5: Schematic of the device orientation in the deposition chambers, where $\theta$ is the angle at which the device is tilted.

\subsubsection{Deposition techniques}

Physical vapor deposition (PVD) techniques, namely thermal evaporation and radiofrequency $(\mathrm{RF})$ sputtering, were utilized in this study to produce uniform thin films. A thermal evaporator is a vacuum deposition technique which utilizes electrical current to heat the desired target, allowing it to vaporize and adhere on the substrate surface [36], as shown in Figure 3.6a. This technique was used for the deposition of $\mathrm{Cr}, \mathrm{Ag}$, and $\mathrm{Au}$ at rates of $0.3 \AA / \mathrm{s}$.

On the other hand, RF sputtering is a surface bombardment technique where non-reactive ions collide with the surface particles of a target, ejecting the target atoms which eventually settle on the substrate. The chamber operates under vacuum conditions, and is filled with sputtering gas (Ar). Power is then supplied to generate $\mathrm{Ar}^{+}$, ions which are directed at the target, ejecting target molecules, which in turn are directed at the substrate, as shown in Figure 3.6b [36]. Sputtering was used to deposit $\mathrm{SiO}_{2}$ thin films at a rate of $0.3 \AA / \mathrm{s}$.

\subsubsection{Thin film structure}

Different thin film structures were chosen and tested to obtain smooth, highly reflective mirrors. The chromium adhesion layer thicknesses were systematically varied between 10 and $20 \mathrm{~nm}$ to deposit smooth films. The most commonly used 
(A)

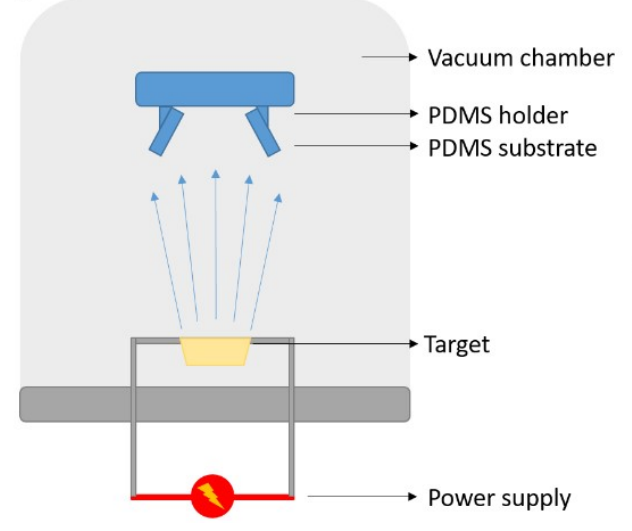

(B)

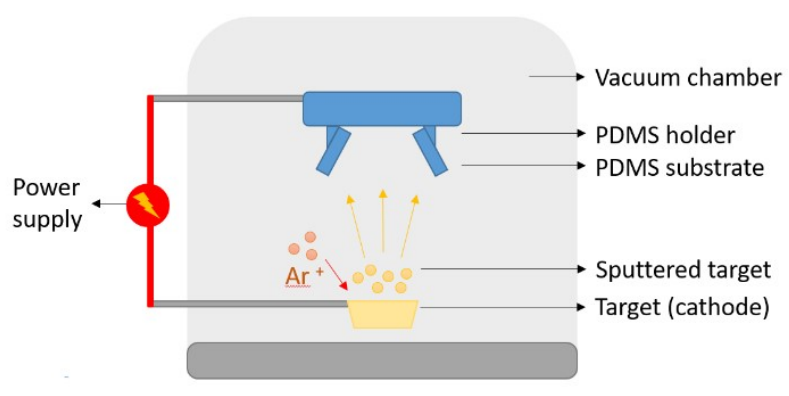

Figure 3.6: Thermal evaporation and sputtering using tilted sample configuration.

mirror materials in the visible wavelength range are aluminum $(\mathrm{Al})$, gold $(\mathrm{Au})$, and silver $(\mathrm{Ag})$, which has the best performance [37].

Silver mirrors have the best reflectance performances at the desired wavelength, however, they form oxide layers, which render them unstable under ambient conditions [38]. Since the oxide layer on a silver film reduces the mirror performance [39], the extent of oxidation of bare silver films after exposure to oxygen plasma was studied in X-ray photoelectron spectroscopy (XPS). Moreover, due to the oxidation of silver, the performances of thin films of Ag covered with other materials were investigated. Therefore, gold, silver, and protected silver films were explored, and the film thicknesses were varied to obtain optimal results. The protective layers tested were gold and $\mathrm{SiO}_{2}$.

Au films. The first tested films were films of $\mathrm{Cr}$ and $\mathrm{Au}$, where the thicknesses of $\mathrm{Cr}$ and $\mathrm{Au}$ were varied and their reflectance values were measured. As the thickness of $\mathrm{Cr}$ enhances the smoothness of the film and hence the reflectance, Cr thicknesses of 10 and $15 \mathrm{~nm}$ were tested with $40 \mathrm{~nm}$ thick gold films. To determine the $\mathrm{Cr}$ thickness that gives the smoothest film, the reflectance values of the mirrors were tested, and the highest reflective mirror was assumed to have the best thickness of $\mathrm{Cr}$, and was used throughout the study. Then, the best Cr thickness was tested with 80 and $150 \mathrm{~nm}$ thick gold films to determine the 
optimum gold thickness.

Ag films. As silver has a higher reflectance performance than Au, Ag films were also considered as mirror materials. To address the oxidation problem of Ag, a protective layer of $\mathrm{Au}$ or $\mathrm{SiO}_{2}$ as shown in Figure 3.7 was tested. As Au coated Ag films are expected to have lower reflectance values than bare Ag films due to the absorbance of $\mathrm{Au}$, the performances of bare Ag films were also explored.

To investigate the thin film performance of $\mathrm{Ag}$ as a mirror, $\mathrm{Cr}-\mathrm{Ag}-\mathrm{Au}$ and $\mathrm{Cr}$ $\mathrm{Ag}_{-} \mathrm{SiO}_{2}$ films were prepared with varying the thicknesses of $\mathrm{Ag}$ films. An overall increase in the reflectance value with increasing silver film thickness is expected. First, Cr-Ag-Au combination was investigated, where 40, 80, and $100 \mathrm{~nm}$ of $\mathrm{Ag}$ were deposited on $\mathrm{Cr}$, and coated with $15 \mathrm{~nm}$ of $\mathrm{Au}$.

Finally, dielectric coating of Ag films was also studied, where the protected Ag films are expected to have similar reflectance values as compared to bare $\mathrm{Ag}$ films. 70, and $80 \mathrm{~nm}$ of $\mathrm{Ag}$ were deposited on $\mathrm{Cr}$ and coated with $15 \mathrm{~nm}$ of $\mathrm{SiO}_{2}$. The expected trend is an increase in reflectance values with increasing silver film thickness.
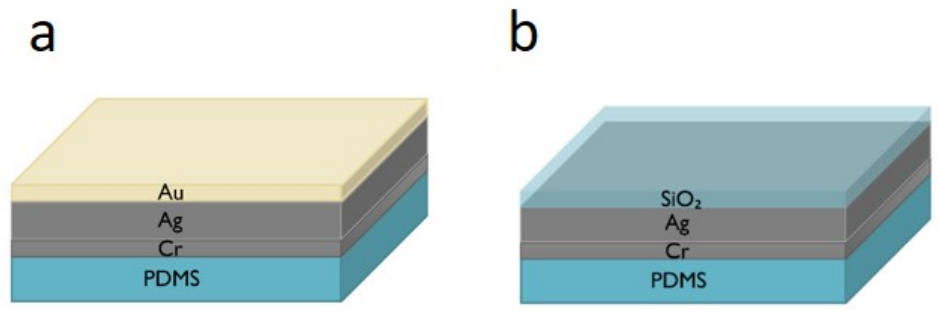

Figure 3.7: Combinations of layered structures tested for Ag thin films for application as mirrors. 


\subsubsection{Mirror characterization}

\subsubsection{Thickness measurements}

In order to ensure that the desired film thicknesses were obtained, a quartz sample was placed in the deposition chambers, and was taped on the sample holders. Once the deposition was complete, the tape was removed from quartz, uncovering a bare quartz region nearby the deposited thin film region. The difference in height between the two regions was investigated using stylus profilometry. A stylus profilometer contains a sample holder, a mechanical probe, and a detector. The mechanical probe in contact with the surface scans the surface, and changes in the probe height give the thickness of the deposited thin film [40].

\subsubsection{Oxidation measurements}

As mentioned earlier in this chapter, the formation of oxide layers on thin silver films causes a reduction in their performance as mirrors. The extent of the silver oxidation was studied using X-ray Photoelectron Spectroscopy (XPS). XPS is a surface analysis technique based on the photoelectric effect, where electrons are ejected when a photon is incident on the surface of a material. Using this phenomena, XPS utilizes monochromatic x-rays in order to generate photoelectrons, and measures their kinetic energies to obtain information about the chemical composition, empirical formula, as well as the electronic structure of the surface [41].

The photoelectric effect occurs when the frequency of the photon is equal to or greater than the threshold frequency required to eject an electron. When the frequency of the photons is above the threshold frequency, electrons are ejected, and the number of ejected electrons depends on the intensity of the incident light. The kinetic energy of the ejected electron is measured in order to calculate the binding energy of the ejected electron, which would be equal to the difference of the incident photon energy and the kinetic energy [42]. 
Thermo Scientific X-ray photoelectron spectrometer with a monochromatic AlK $\alpha$ source was used to measure the amount of surface oxidation. The sample surfaces were irradiated with a pass energy of $187.5 \mathrm{eV}$, and the measurements were obtained from three different points on the surface with at least two sweeps per data point. The binding energy range used for chemical identification was 0-1100 eV. Peak positions recorded in the Handbook of XPS were used as the reference standard for spectra identification and data interpretation.

\subsubsection{Roughness measurements}

Another important parameter that affects the performance of the films as mirrors is roughness, as smoother films yield higher reflective surfaces [43]. For this reason, roughness data was collected for the final films using atomic force microscopy (AFM). This instrument uses the interaction between a cantilever tip and the sample surface in order to study the sample's surface properties. An AFM is generally composed of a cantilever tip and an optical system which detects the deflections of the tip. Roughness measurements are carried out by detecting the deflection of the cantilever in the z-axis in tapping mode, where the cantilever tip oscillates at amplitudes of 100-200 nm, and gently taps the surface as it scans it [44].

\subsubsection{Reflectance measurements}

In order to quantify the performance of the fabricated thin films as mirrors, thin films were deposited on the channel walls as well as on flat quartz and PDMS pieces. The reflectance was measured using a white light reflectance spectroscopy (WLRS) set-up. The mirror under study was irradiated by a Xenon lamp source, and the back reflected photons were detected using a reflection probe connected to Maya2000 Pro spectrometer with an optical fiber. The reflectance value of the mirror was then calculated by comparing the intensity of back reflected photons to a $100 \%$ reflective mirror reference. 


\subsection{Microfluidic device operation}

\subsubsection{Chemical compatibitily}

The CQWs used in this study are most commonly dissolved in the organic solvents toluene and hexane, which are highly capable of swelling PDMS. In this section, PDMS swelling and enhancement are discussed.

In 2003, PDMS compatibility with several solvents was studied by Lee et al. [45]. Their findings classify toluene and hexane as "high PDMS solubility solvents", and render them as incompatible for use with PDMS devices. In their study, Lee et al. investigate the swelling behavior of PDMS bonded to glass, and state that the stress exerted on PDMS cause it to detach from glass, since swelling occurs in PDMS and not in glass. Therefore, it is expected that these solvents would change the channel dimensions.

To account for the changes in the channel cross section caused by swelling, the PDMS was brought to the equilibrium swelling state before use, after which it was not expected to swell further. To overcome the problem of glass detachment, PDMS was bonded to PDMS, allowing equal stresses on both the channel components, therefore preventing uneven stresses which lead to unsealing [45].

In 2014, Hidrovo et al. [46] proposed improving the PDMS material to make it suitable for use with organic solvents. Their method involved enhancing the PDMS performance by increasing its stiffness, and was therefore simple and introduced no new materials which require post processing. Their method combined the previous works by Fuard et al. [47] and Park et al. [48], who used thermal aging, and increased the ratio of curing agent to PDMS, respectively. Hidrovo et al. studied the effects of thermal treatment and curing agent combined.

The study showed that the best results were obtained when a ratio of 5:1 PDMS to curing agent was used, and thermal treatment of the devices at $200^{\circ}$ for 24 hours was carried out. This method is further proven by Hidrovo et al. to 
reduce the swelling of PDMS in toluene from 55\% to less than $10 \%$ [46].

\subsubsection{Plasma bonding and operation}

After mirror deposition on the microfluidic devices, the pattern-holding PDMS layers were sealed with flat PDMS layers using oxygen plasma, which is an ionized gas generated by exposing neutral gas to radio frequency power. Plasma contains free electrons, ionized gas, and radicals, which heat, sputter, and etch the surface of a sample, respectively. These three basic phenomena make plasma an effective way to clean and activate surfaces.

Electrons heat the surface of the substrate and remove the physiosorbed, or weakly bonded, contaminants. Ionized gas bombards the surface due to applied voltage between the gas and the sample, resulting in a non-selective sputtering effect. The most effective surface cleaning factor in plasma is etching due to radicals which react with the substrate's surface. Harric plasma cleaner (PDC$32 \mathrm{G}$ ) was used in this work.

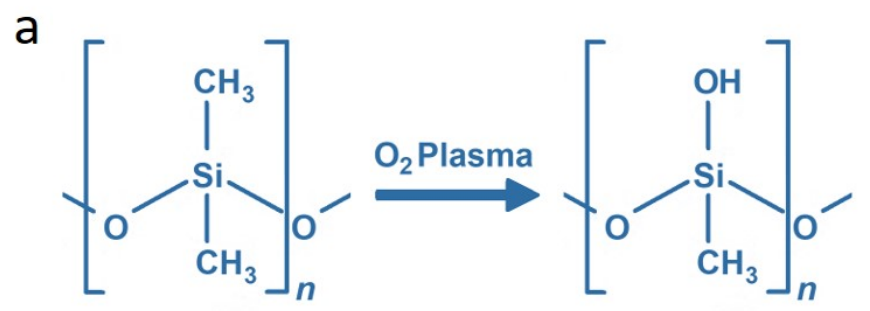

b

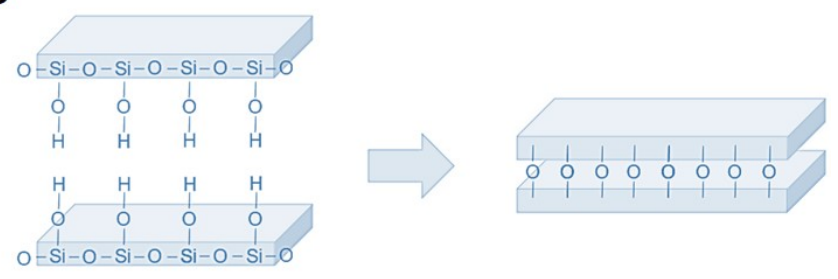

Figure 3.8: (a) Oxygen plasma activation of PDMS surface, and (b) bonding.

The radicals adsorb on the surface, react, and desorb, and the reaction's byproducts are pumped out of the system. The choice of plasma gas used for 
etching depends on the surface under study. Due to the organic nature of PDMS, oxygen plasma was desired in order to activate the surface bonds, allowing the PDMS substrates to adhere as shown in Figure 3.8 [49].

Two pumping methods were used to achieve flow in the microfluidic channels in this study, namely, passive and active pumping. For passive pumping, or capillary driven flow (CDF), flow was achieved by simply introducing fluid into the inlet of the channel. When this method was used, flow focusing was not achieved, and the whole channel width was used as the gain medium. For active pumping, or pressure driven flow (PDF), syringe pumps were used. When this method was used, flow focusing was achieved, and the width occupied by CQWs was tunable by varying the width of the soft wall created by water.

\subsubsection{Contact angle measurements}

The contact angle was measured in order to gain an understanding of whether or not capillary forces could induce flow in the channels. For the solution to fill the channel spontaneously, the contact angle must be small enough to induce a pressure drop, as shown in Equation 2.27. Low contact angles are associated with higher wetting solvents, and result in flow.

As toluene was the carrying medium of the CQWs used for the capillary driven experiments, the contact angle of toluene on a treated PDMS surface was measured. Static contact angle measurement technique was employed using a contact angle goniometer, shown in Figure 3.9.

First, a PDMS sample was prepared by mixing it with curing agent at a ratio of 5:1, then, the PDMS substrate was thermally treated for $24 \mathrm{~h}$ on a hot plate at $200^{\circ} \mathrm{C}$. The PDMS sample was then placed on a goniometer, and the toluene droplet was released from a syringe positioned above the PDMS surface. A high resolution camera then captured the profile of the droplet on the sample surface, and the contact angle was then measured from the image. 


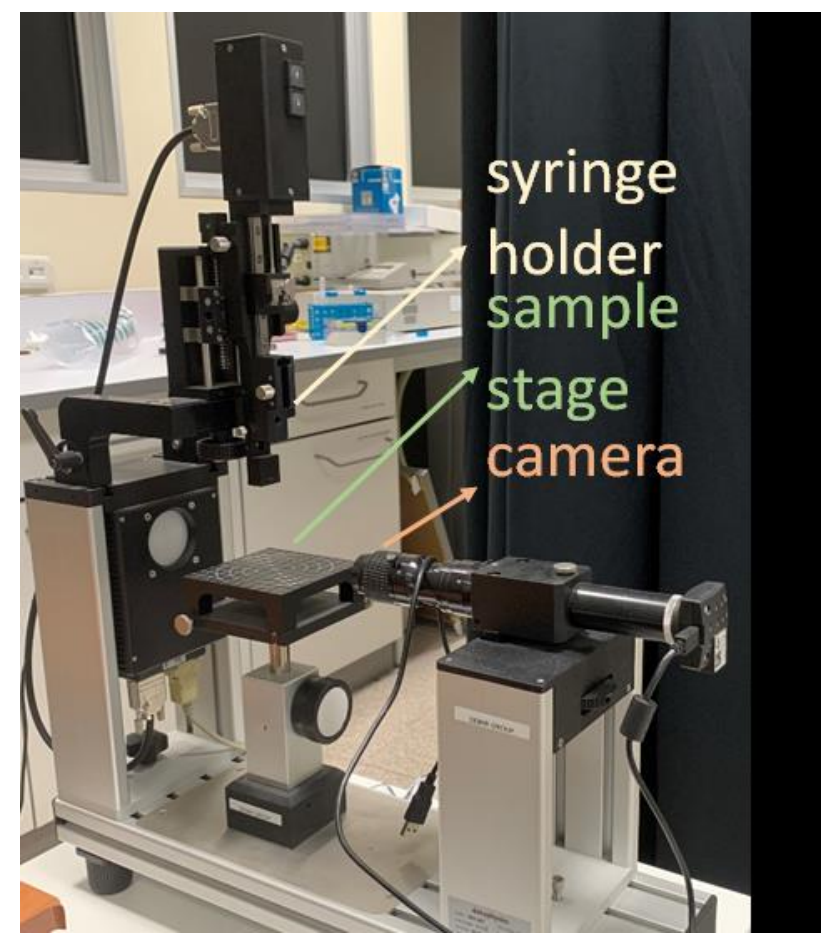

Figure 3.9: Contact angle goniometer.

\subsection{Colloidal semiconductor nanocrystal char- acterization}

\subsubsection{Absorption spectroscopy}

Absorption spectroscopy is an analytical method that is used to characterize materials and analyze their concentrations. It employs the basic principle of photon absorption. When the energy gap of a material is equal to that of the incident photon, the photon is absorbed. In absorption spectroscopy, photons of different wavelengths are incident on the material, and an absorption spectrum is generated based on the energy gaps present in the material.

Light from a broadband light source is directed onto a rotating diffraction grating which splits it into several beams. Light is then directed onto a slit, or an 
aperture, that selects the desired wavelengths. This system acts as a monochromator, and by rotating the diffraction grating, it allows multiple excitation wavelengths. The steady state absorption spectroscopy is demonstrated in Figure 3.10 .

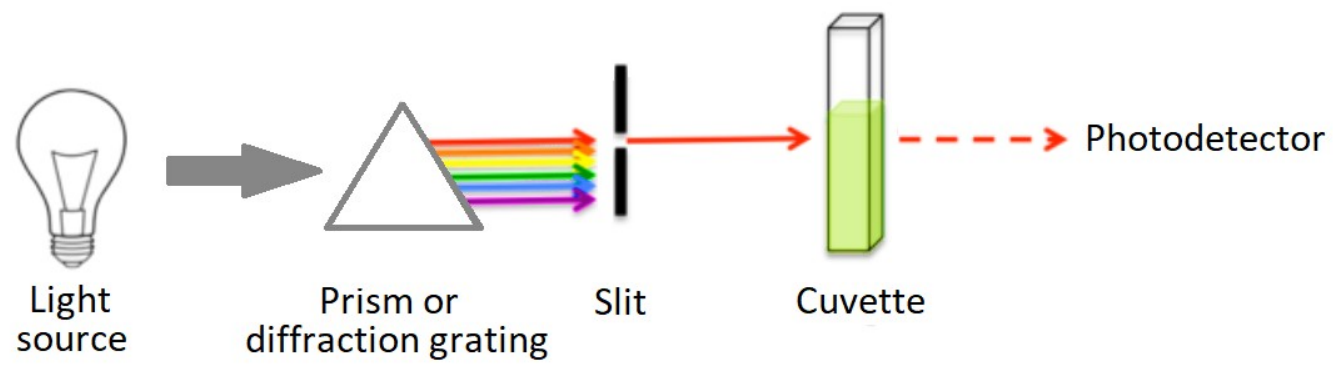

Figure 3.10: Absorption spectroscopy.

According to Beer-Lambert's law shown below, absorbance is a function of sample concentration, c, the distance the light travels inside the cuvette, L, and the molar extinction coefficient, $\epsilon$.

$$
A=\epsilon c L
$$

The analyzed samples contain CQWs dissolved in toluene or water, and a blank toluene or water sample was used as a reference. However, when light strikes onto CQWs, some of it may also be scattered or reflected. To prevent the undesired scattering and reflection, low concentrations of CQWs were used.

Absorption was also used to analyze the thickness of the CQWs, as their optical properties are highly dependent on their thickness. Moreover, the formation of hetero-structures may also be detected by red and blue shifts in the absorption spectrum.

\subsubsection{Photoluminescence spectroscopy}

Another important characterization of colloidal nanocrystals is photoluminescence spectroscopy. This technique was used to measure the spontaneous emission 
from nanocrystals following optical excitation. As the emission and absorption characteristics of nanocrystals are size-dependent, PL spectroscopy determines the thickness of the CQWs. The experimental set-up for this measurement consists of a monochromator as the one used in the absorption spectroscopy in order to excite the sample, and another monochromator to collect the emission.

\subsubsection{Electron microscopy}

Surface imaging is another important characterization technique for CQWs, as it provides an accurate platform to measure their dimensions. Optical microscopes magnify matter using light refractions, therefore, the wave properties of light limit their resolution. Instead, scanning electron microscopes (SEMs) and tunneling electron microscopes (TEMs), which use electron beams rather than electromagnetic radiation to scan the surfaces, were utilized for the imaging of CQWs [50].

In SEM, the incident electron beam on the material stimulates the emission of backscattered electrons, secondary electrons, and x-rays emitted from the surface. The sample image is then generated by collecting the secondary electrons emitted from the sample. In TEM, the electron beam is incident on an ultrathin layer of specimen, and an image is then generated by collecting the transmitted electrons.

Therefore, while detailed morphology and surface topology of a specimen may be obtained from SEMs, TEMs have the advantage of providing details about the internal structures of the specimen such as its crystallinity [50]. Both SEM and high angle annular dark filed TEM (HAADF-TEM) were used to analyze the CQWs used in this work. 


\subsection{Optical gain measurements}

The measurement of optical gain of CQWs is necessary in order to determine their lasing characteristics. As mentioned earlier, amplified spontaneous emission (ASE) occurs when the gain is greater than loss, in other words, when population inversion is achieved. In order to initiate optical gain, CQWs were pumped with a pulsed laser as an optical pumping source. The pump fluence was gradually increased until ASE was observed.

The optical set-up used for the experiments consists of a pump laser, an attenuator to adjust the intensity, a Barium Borate (BBO) crystal, a cylindrical lens, a short path filter, and a fiber connected to a spectrometer, as shown in Figure 3.11. A pulsed Spectraphysics laser at $800 \mathrm{~nm}$, with $120 \mathrm{fs}$ pulse width and $1 \mathrm{kHz}$ repetition rate was used as the pump. The sample was mounted on a stage with adjustable $\mathrm{x}, \mathrm{y}$, and $\mathrm{z}$ translations. Moreover, the power of the incident beam was measured using a power meter by using a beam splitter.

The $800 \mathrm{~nm}$ laser beam was used for one-photon and two-photon absorption regimes. In this thesis, sample excitation in the one-photon absorption regime was studied. For one-photon absorption, the laser beam was passed through a BBO (beta barium borate) crystal to double its frequency. Using a bandpass filter, the remaining $800 \mathrm{~nm}$ beam was filtered out. Before the $\mathrm{BBO}$ crystal, the pump intensity was adjusted using an attenuator, or a rotatable neutral density (ND) filter. Since a stripe shaped beam was desired for our experiments, a cylindrical lens was used. The emission from the sample was collected by an optical fiber connected to a spectrometer (Avantes).

To study the gain performance in microfluidic channels, devices were fabricated and a solution of CQWs in toluene was first prepared. The CQWs were then introduced in the channel, while the microfluidic devices were mounted on the sample holder as shown in the inset of Figure 3.11. The sample was then optically pumped with gradually increasing pump intensity, and its emission was collected. It is expected to observe ASE from the devices above a threshold pump intensity, 


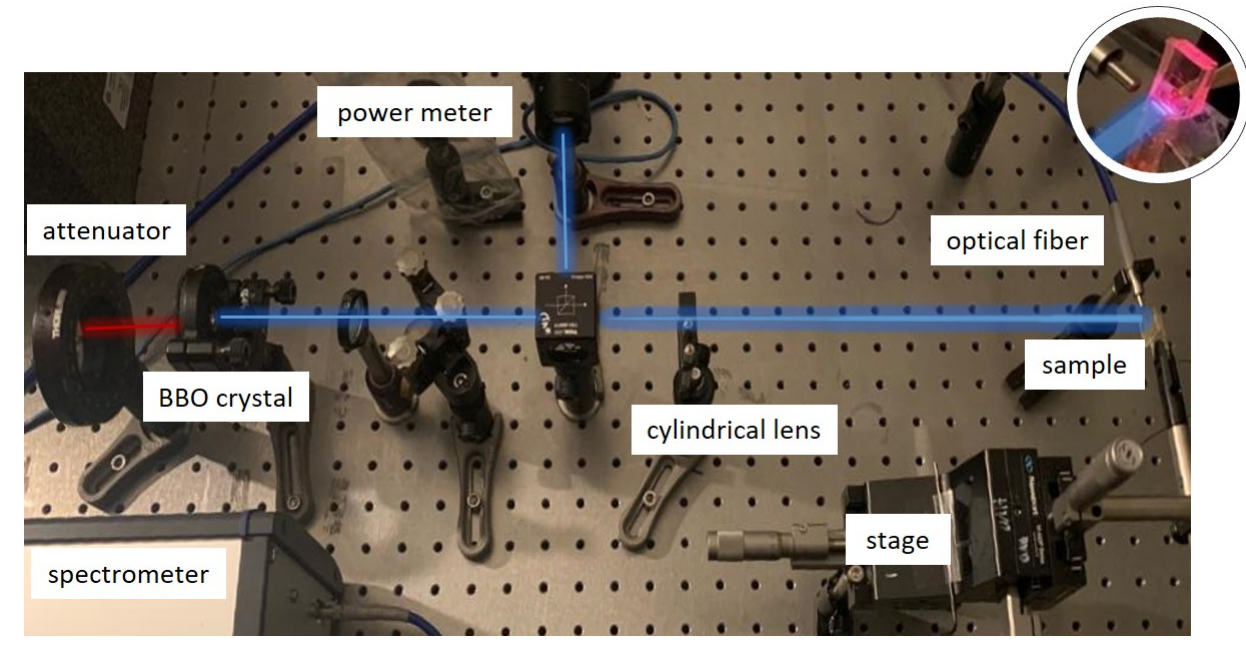

Figure 3.11: Optical pumping and collection setup for gain measurements under one-photon absorption.

and lasing from devices with an optical cavity, due to the optical feedback that the mirrors provide. 


\section{Chapter 4}

\section{Optical gain and lasing in microfluidic devices}

\subsection{Motivation}

In this thesis work, microfluidic CQW waveguides and lasers were created in PDMS elastomer chips to provide inexpensive and compact coherent light sources for numerous applications in integrated optics and microfluidics. Toluene soluble CQWs were dissolved in toluene and introduced to the channels using capillary forces, and aqueous CQWs were introduced to the channels by the application of a pressure gradient to achieve in-solution optical gain. Capillary filled microfluidic devices provide ease of operation on the expense of the ability to reconfigure the waveguide or cavity parameters during operation. In pressure driven devices, the width of the gain medium can be controlled by controlling the flowrates of the core and cladding streams. Moreover, the pressure driven devices offer the possibility to create a tunable in-solution white laser beam by flowing 3 independent streams of red, green, and blue CQWs in the same optical cavity in a laminar fashion 


\subsection{Sample preparation}

\subsubsection{CQWs synthesis}

4ML CdSe/CdS@ 3 ML $\mathrm{Cd}_{1-x} \mathrm{Zn}_{x} \mathrm{~S}$ core/crown@shell CQWs and $4 \mathrm{ML}$ CdSe/CdS@4 ML CdZnS core/crown@shell CQWs were synthesized for this work. The synthesis follows a previously reported recipe by Shendre et al with slight modifications [51]. The CQWs were synthesized by first preparing the desired number of monolayers of CdSe core, then growing CdS crown, and finally growing the desired number of shells to achieve the desired hetero-structue. The synthesis procedure is provided in this section, and detailed recipe may be found in the previously published work.

\subsubsection{CdSe core synthesis}

Cadmium myristate was prepared by mixing two solutions of cadmium nitrate tetrahydrate and sodium myristate. The cadmium nitrate solution was prepared by dissolving $1.23 \mathrm{~g}$ in $40 \mathrm{~mL}$ methanol, and the sodium myristate solution was prepared by dissolving $3.13 \mathrm{~g}$ in $250 \mathrm{~mL}$ of methanol. The solutions were stirred for a few hours, and the cadmium myristate precipitate was filtered out of the solution, washed with methanol, and left to dry for 12 hours in vacuum.

In a three neck flask, $170 \mathrm{mg}$ of the prepared cadmium myristate was mixed with $12 \mathrm{mg}$ of selenium powder and $15 \mathrm{~mL}$ of 1-Octadecene (ODE). The solution was then degassed at $90^{\circ} \mathrm{C}$ and heated in an argon environment at $240^{\circ} \mathrm{C}$. $90 \mathrm{mg}$ of cadmium acetate dehydrate was added to the flask when the solution color changed to deep orange at around $190-200^{\circ} \mathrm{C}$. For CQW growth, the temperature of the solution was kept at $240^{\circ} \mathrm{C}$. After 8 minutes, the reaction was finally quenched in a water bath, and $0.5 \mathrm{~mL}$ of oleic acid was added to the flask. The 4 ML CdSe core was then precipitated by adding acetone and redispersing in hexane. Size selective precipitation in ethanol was carried out in order to remove undesired CQWs. The precipitation was achieved by adding a few drops of 
ethanol to the solution, centrifuging, and collecting the precipitate.

\subsubsection{CdSe/CdS core/crown synthesis}

A CdS crown growth solution was prepared for the crown growth. The growth solution was prepared by mixing $480 \mathrm{mg}$ of cadmium acetate dehydrate and 340 $\mu \mathrm{L}$ of oleic acid in $2 \mathrm{~mL}$ of ODE. This mixture was sonicated for 30 minutes and then heated to $160^{\circ} \mathrm{C}$ on a hot plate with continuous stirring. Once a homogenous white gel was obtained, $4 \mathrm{~mL}$ of $0.1 \mathrm{M}$ S-ODE solution was added to the mixture.

The core/crown synthesis was carried out in a three neck flask, in which the synthesized $4 \mathrm{ML}$ CdSe solution in hexane was loaded with $15 \mathrm{~mL}$ of ODE. The flask was left under vacuum conditions and heated to $80^{\circ} \mathrm{C}$ where hexane was removed. The flask was then heated to $240^{\circ} \mathrm{C}$ in argon gas, and $\mathrm{CdS}$ growth solution was injected at a rate of $15 \mathrm{~mL} / \mathrm{h}$. The reaction was quenched in a water bath once the desired crown size was reached, and $10 \mathrm{~mL}$ of hexane was added. Unreacted compounds were removed by precipitating the mixture in ethanol. The precipitated CQWs were re-dispersed in hexane and washed again with ethanol to ensure the removal of undesired species.

\subsubsection{CdZnS core/crown@shell synthesis}

The core/crown@shell structure was synthesized by colloidal atomic layer deposition (c-ALD) on the previously prepared core/crown CQWs. The CdSe/CdS core/crown CQWs were dispersed in $1 \mathrm{~mL}$ hexane and mixed with $4 \mathrm{~mL} \mathrm{~N}$ methylformamide (NMF) and 40-48\% aqueous ammonium sulfide solution, which acts a precursor for the sulfur shell growth. The solution was mixed for 2 minutes, and the shell growth was quenched by adding $1 \mathrm{~mL}$ acetonitrile and an excess amount of toluene until the mixture became cloudy. The mixture was then precipitated by centrifugation, re-dispersed in NMF, and precipitated again to get rid of unreacted species. For 4ML CdSe/CdS@ $3 \mathrm{ML} \mathrm{Cd}_{1-x} \mathrm{Zn}_{x} \mathrm{~S}$ core/crown@shell CQWs, a cation precursor mixture was prepared by mixing two solutions of 0.4 
$\mathrm{M} \mathrm{Cd}\left(\mathrm{NO}_{3}\right)_{2} \cdot 4 \mathrm{H}_{2} \mathrm{O}$ and $0.4 \mathrm{M} \mathrm{Zn}\left(\mathrm{NO}_{3}\right)_{2} \cdot 6 \mathrm{H}_{2} \mathrm{O}$ in $\mathrm{NMF}$ with a volume fraction of $5 \%$ and $95 \%$, respectively.

The CQWs precipitate was then re-dispersed in $4 \mathrm{~mL}$ of NMF and mixed by stirring with $1 \mathrm{~mL}$ of cation precursor for 45 minutes. To get rid of unreacted species, the cleaning steps were repeated, and the c-ALD steps described in this section were repeated to increase the number of shells. Finally, the CQWs were precipitated and stirred with $5 \mathrm{~mL}$ of hexane and $100 \mathrm{~mL}$ of oleic acid for 12 hours. The CQWs were then precipitated in ethanol to remove excess ligands, and stored in toluene.

The synthesis of $4 \mathrm{ML} \mathrm{CdSe} / \mathrm{CdS} @ 4 \mathrm{ML} \mathrm{CdZnS}$ core/crown@shell CQWs follows the same recipe, with the volume fractions of the cation precursor mixtures of $0.4 \mathrm{M} \mathrm{Cd}\left(\mathrm{NO}_{3}\right)_{2} \cdot 4 \mathrm{H}_{2} \mathrm{O}$ and $0.4 \mathrm{M} \mathrm{Zn}\left(\mathrm{NO}_{3}\right)_{2} \cdot 6 \mathrm{H}_{2} \mathrm{O}$ in NMF were 50:50, 5:95, 5:95, and 1:99 for the first, second, third, and fourth layers, respectively.

\subsubsection{Ligand exchange}

The 4 ML CdSe/CdS@ 4 ML CdZnS core/crown@shell CQWs used in this study were dispersed in water for pressure-driven experiments. For this reason, ligand exchange was performed as described in this section. The CdSe/CdS@CdZnS core/crown@shell CQWs (synthesized form 5OD(@370nm) core/crown) in hexane were precipitated with ethanol and dispersed in $3 \mathrm{~mL}$ chloroform. $1 \mathrm{~mL}$ of EDA (ethylene diamine) was then added and stirred for an hour, followed by the addition of $3 \mathrm{~mL} 0.15 \mathrm{M} \mathrm{MPA}$ (3-mercaptopropionic acid) solution in water. As CQWs moved to the water phase, chloroform was removed with a pipette. Finally, 10mg of $\mathrm{Zn}\left(\mathrm{NO}_{3}\right)_{2} \cdot 6 \mathrm{H}_{2} \mathrm{O}$ was added to the CQW dispersion in water, and the solution was stored. 


\subsubsection{Microfluidic device preparation and optimization}

The layout of the microfluidic devices was created by using Tanner L-edit program, and then transferred to a dark field glass mask. The design from the glass mask was transferred to a silicon wafer using the photolithography technique, and finally to a PDMS substrate using the soft lithography technique as described earlier in Section 3.1. A summary of the processes is shown in Figure 4.1 .

The devices were prepared at 5:1 PDMS to curing agent ratio and treated at $200^{\circ} \mathrm{C}$ for 24 hours. For the FP cavity devices, the mirrors were deposited as described in Section 3.2.1, prior to sealing the PDMS devices. The film compositions were chosen according to the combinations that give the best reflectance performances.

The same devices were intended for use in both capillary and pressure driven flows. For this reason, inlets for the core and the cladding fluids were designed. Depending on the operation method, the cladding stream inlet of each device may or may not be used. For capillary driven flows, only the inlets for CQWs were required, and thus the cladding inlets were rendered passive by keeping them closed, as capillary filling only occurs when air in the channels can be displaced and expelled out of the channels by the gain material.

Flow focusing of CQWs in the micro-channels was desired in order to create soft walls of fluid flowing past one another in a laminar fashion for applications in reconfigurable light sources. A solution of methylene-blue in water was allowed to flow in the microfluidic devices as the focused stream, and water as the cladding stream. Methylene-blue solution and water pair were chosen for this experiment in order to experimentally study the width of the focused stream as a function of varying flowrates and to compare it to the expected trend in Equation 2.24.

Finally, to check the feasibility of capillary flow of CQWs in the channels, the contact angle of treated PDMS and toluene was measured using a contact angle goniometer. The pressure drop was calculated as in Equation 2.27, using the 


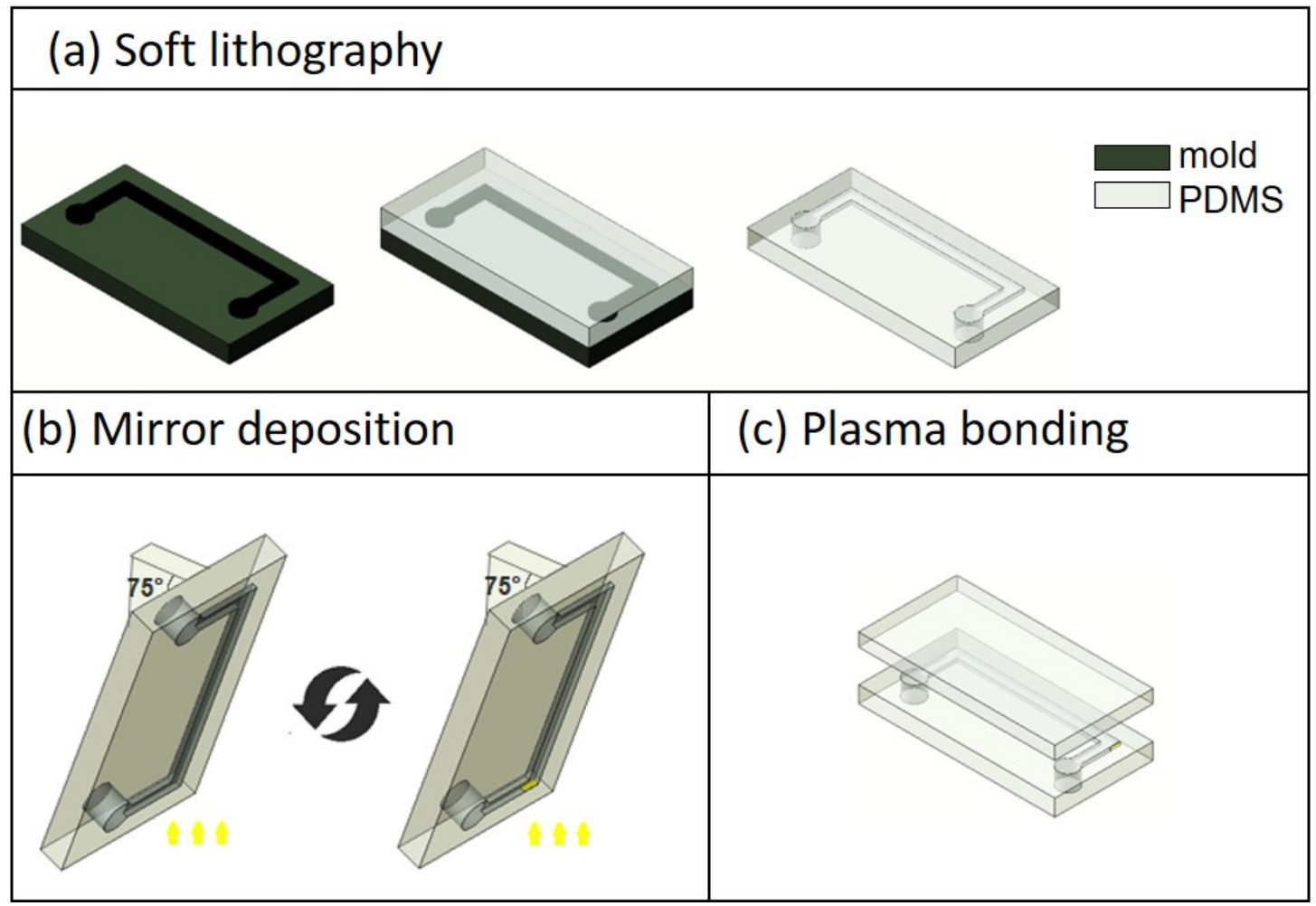

Figure 4.1: Schematic illustration of our device fabrication steps.

measured contact angle.

\subsection{Results and discussion}

\subsubsection{CQW characterization}

The 4ML CdSe/CdS@ 3 ML $\mathrm{Cd}_{1-x} \mathrm{Zn}_{x} \mathrm{~S}$ core/crown@shell CQWs and $4 \mathrm{ML}$ CdSe/CdS@4 ML CdZnS core/crown@shell CQWs characterization are presented in this section. Absorption spectroscopy, PL spectroscopy, SEM, and TEM imaging were carried out for the CQWs. The PL and absorption spectra, TEM images, and SEM imagse of 4ML CdSe/CdS@ $3 \mathrm{ML} \mathrm{Cd}_{1-x} \mathrm{Zn}_{x} \mathrm{~S}$ core/crown@shell CQWs are shown in Figures 4.2a-c, respectively, and of 4 ML CdSe/CdS@ 4 ML CdZnS core/crown@shell CQWs are shown in Figures 4.2d-f, respectively. 
Optical characterization results of $4 \mathrm{ML}$ CdSe/CdS@ $3 \quad \mathrm{ML} \mathrm{Cd}_{1-x} \mathrm{Zn}_{x} \mathrm{~S}$ core/crown@shell CQWs are reported in Figure 4.2a, which shows the photoluminescence of the CQWs centered at $633 \mathrm{~nm}$ with a linewidth of $30 \mathrm{~nm}$. The transition is observable in the absorption spectrum, where the upward heavy-hole peak is evident at $626 \mathrm{~nm}$, and the light-hole transition at $575 \mathrm{~nm}$. These CQWs are $3.2 \mathrm{~nm}$ thick, and have lateral dimensions of 26 by $14 \mathrm{~nm}^{2}$.

Similarly, the optical characterization results of $4 \mathrm{ML}$ CdSe/CdS@ $4 \mathrm{ML}$ CdZnS core/crown@shell CQWs are reported in Figure 4.2d. The photoluminescence of these CQWs is centered at $635 \mathrm{~nm}$ with a linewidth of $23 \mathrm{~nm}$. The upward heavy-hole and the light-hole transition peaks are located at $633 \mathrm{~nm}$ and $580 \mathrm{~nm}$, respectively. The lateral dimensions of these CQWs are 26 by $14 \mathrm{~nm}^{2}$, and their thicknesses are $3.8 \mathrm{~nm}$.
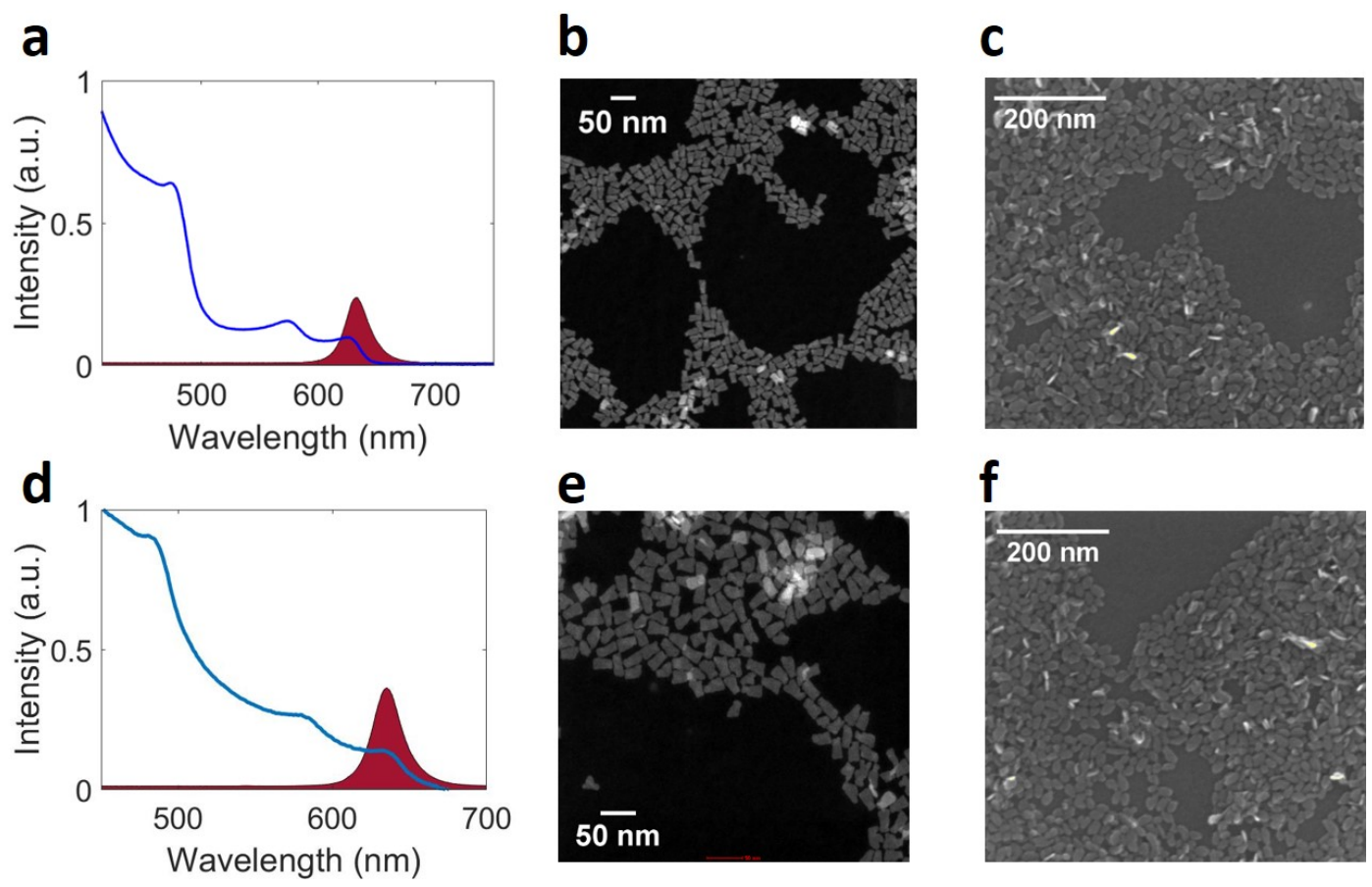

Figure 4.2: (a) Photoluminescence (red) and absorption (blue) spectra, (b) TEM image, and (c) SEM image of $4 \mathrm{ML} \mathrm{CdSe} / \mathrm{CdS} @ \mathrm{Cd}_{1-x} \mathrm{Zn}_{x} \mathrm{~S}$ core/crown@ $3 \mathrm{ML}$ shell CQWs. (d) Photoluminescence (red) and absorption (blue) spectra, (e) TEM image, and (f) SEM image of 4 ML CdSe/CdS@CdZnS core/crown@ 4 ML shell CQWs. 


\subsubsection{Flow focusing and capillary flow}

To test the effect of flowrates on the width of the focused stream, water was allowed to flow at a constant flowrate of $10 \mu \mathrm{L} / \mathrm{min}$, and the flowrate of methyleneblue solution was increased from $5 \mu \mathrm{L} / \mathrm{min}$ to $20 \mu \mathrm{L} / \mathrm{min}$ by increments of 5 $\mu \mathrm{L} / \mathrm{min}$. The ratio of width of the focused stream to the full channel width was plotted as a function of the ratio of the volumetric flowrate of the focused stream to the total volumetric flowrate, as shown in Figure 4.3a.

While the expected trend in was a linear relationship with a slope of 1 , the resulting slope is 0.99. This result confirms the trend predicted in Equation 2.24, and the deviation in the results is accounted for experimental errors. The flow profiles shown in Figure 4.3b from left to right represent dye to water flowrates of 5:10, 10:10, 15:10, and 20:10 $\mu \mathrm{L} / \mathrm{min}$ (dye flowrate: water flowrate).

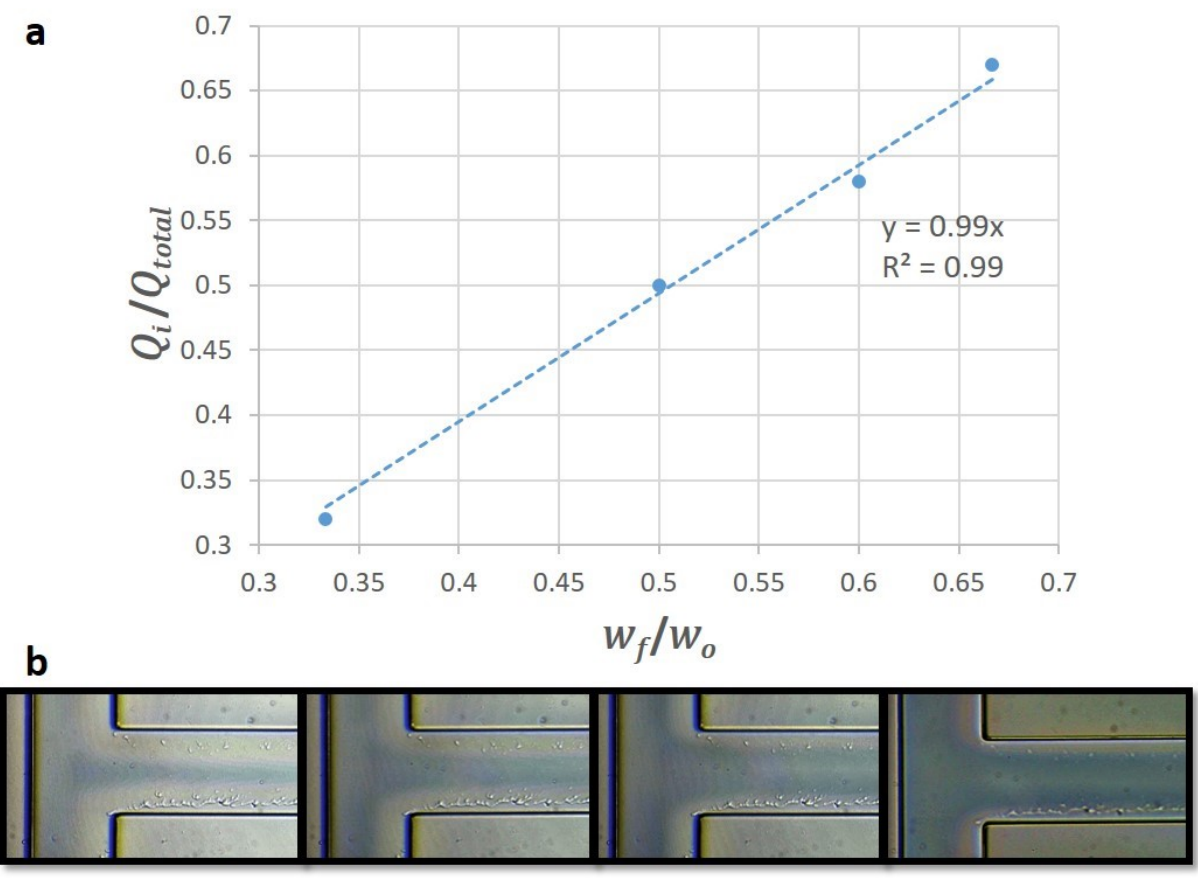

Figure 4.3: (a) Shows the ratio of the width of the focused stream to the total width of the channel as a function of the ratio of the volumetric flowrate of the focused stream to the total volumetric flowrate (b) Images of the focused streams at methylene blue volumetric flowrates of $5,10,15$, and $20 \mu \mathrm{L} / \mathrm{min}$, from left to right. 
Introducing CQWs in toluene to the channel inlet resulted in observable capillary flow, suggesting that the capillary forces were strong enough between the solution and PDMS. To justify, the contact angle of toluene on treated PDMS was measured and found to be $30.1^{\circ}$, as shown in Figure 4.4a.

Plugging the result in the Young-Laplace equation for $300 \mu \mathrm{m}$ wide, $100 \mu \mathrm{m}$ deep channels gave a pressure drop of $0.65 \mathrm{kPa}$, meaning that the capillary forces were large enough to induce flow in the channels. As low concentrations of CQWs in toluene were used, it was assumed that toluene containing CQWs would have a similar contact angle. This assumption can be confirmed from Figure 4.4b, which shows a fluorescent microscope image of capillary filled channel.

a

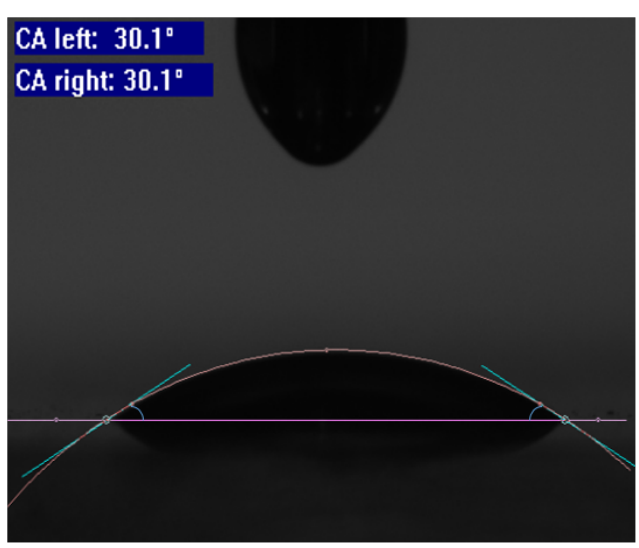

b

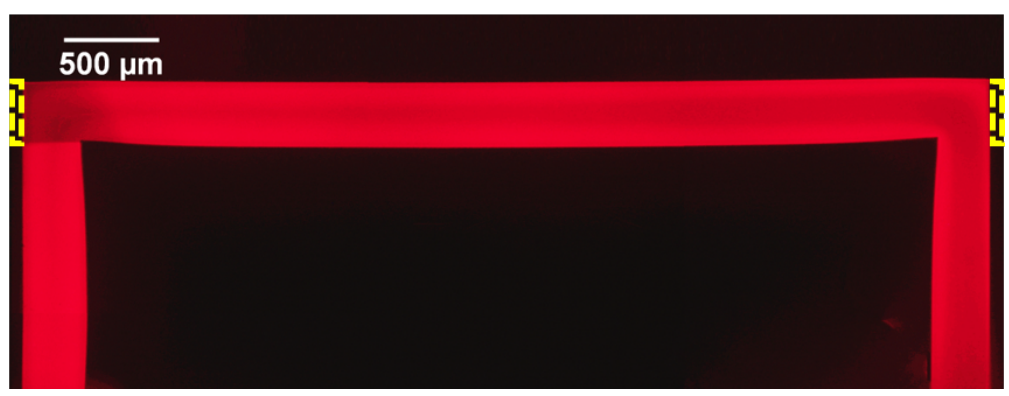

Figure 4.4: (a) Contact angle of toluene on treated PDMS, (b) fluorescent microscope image of capillary filled channel (shown with mirrors marked on the photograph). 


\subsubsection{Mirror quality}

\subsubsection{Mirror reflectance}

Thin films of $\mathrm{Ag}$ and $\mathrm{Au}$ with various combinations and thicknesses were fabricated and thir reflectance values at $650 \mathrm{~nm}$ were measured as described earlier. Stylus profilometer was used after deposition in order to monitor the thicknesses of the deposited films. A summary of the results is shown in Table 2. The 12 mirror combinations tested are numbered, and the thicknesses of the layers are shown in $\mathrm{nm}$. The resulting reflectance values on flat PDMS pieces are recorded.

\begin{tabular}{|c|c|c|c|c|c|c|c|c|c|c|c|}
\hline$\#$ & $\mathrm{Cr}$ & $\mathrm{Ag}$ & $\mathrm{Au}$ & $\mathrm{SiO}_{2}$ & $\% \mathbf{R}$ & $\#$ & $\mathrm{Cr}$ & $\mathrm{Ag}$ & $\mathrm{Au}$ & $\mathrm{SiO}_{2}$ & $\% \mathbf{R}$ \\
\hline \multicolumn{6}{|c|}{$\mathrm{Cr}$ and $\mathrm{Au}$} & \multicolumn{6}{|c|}{$\mathrm{Cr}, \mathrm{Ag}$, and $\mathrm{Au}$} \\
\hline 1 & 10 & & 40 & & 46 & 7 & 15 & 40 & 15 & & 77 \\
\hline 2 & 15 & & 40 & & 54 & 8 & 20 & 40 & 15 & & 81 \\
\hline 3 & 15 & & 80 & & 57 & 9 & 15 & 80 & 15 & & 85 \\
\hline 4 & 15 & & 150 & & 49 & 10 & 15 & 100 & 15 & & 81 \\
\hline \multicolumn{6}{|c|}{$\mathrm{Cr}$ and $\mathrm{Ag}$} & \multicolumn{6}{|c|}{$\mathrm{Cr}, \mathrm{Ag}$, and $\mathrm{SiO}_{2}$} \\
\hline 5 & 15 & 40 & & & 68 & 11 & 20 & 70 & & 15 & 93 \\
\hline 6 & 15 & 80 & & & 89 & 12 & 20 & 80 & & 15 & 95 \\
\hline
\end{tabular}

In combinations 1 and 2, the effect of increasing Cr thickness was tested. A 40 $\mathrm{nm}$ thick film of $\mathrm{Au}$ deposited on PDMS had a reflectance value of $46 \%$ on $10 \mathrm{~nm}$ Cr. The reflectance value jumped from $46 \%$ to $54 \%$ once the $\mathrm{Cr}$ film thickness was increased to $15 \mathrm{~nm}$. This behavior was expected as the roughness of the thin films decreases with increasing Cr thicknesses, and as smoother films yield better mirrors.

The optimum Au film thickness was tested in combinations 2-4, where the performance of 40, 80, and $150 \mathrm{~nm}$ of $\mathrm{Au}$ films were compared. Increasing the gold film thickness from 40 to $80 \mathrm{~nm}$ resulted in a slight increase in reflectance value, and the deposition of $150 \mathrm{~nm}$ of gold resulted in a reflectance value lower than that of $40 \mathrm{~nm}$ gold. The drop in reflectance value at $150 \mathrm{~nm}$ may be a result 
of losses due to the absorption of incident light by the relatively thick $\mathrm{Au}$ film. As the highest reflectance value obtained from Au was 57\%, the use of Ag films was required to enhance mirror performance.

In combinations 5 and 6, 40 and $80 \mathrm{~nm}$ thin films of $\mathrm{Ag}$ were deposited on 15 $\mathrm{nm}$ Cr. These combinations resulted in $68 \%$ and $89 \%$ reflectance values, proving that Ag thin film performance is superior to that of Au. However, the Ag films were kept in an oxygen free environment prior to reflectance measurements, since their reflectance values were expected to decrease upon forming oxide layers. As the mirrors were exposed to oxygen plasma after deposition to seal the devices, it was important to protect the Ag films. Therefore, Ag films were protected by depositing thin films of $\mathrm{Au}$ in combinations 7-10 and $\mathrm{SiO}_{2}$ in combinations 11-12. $15 \mathrm{~nm}$ of protective layers were chosen as thinner films may form islands instead of smooth, uniform films.

In combinations 7, 9, and 10; 40, 80, and $100 \mathrm{~nm}$ of $\mathrm{Ag}$ films were deposited respectively on $15 \mathrm{~nm} \mathrm{Cr}$, followed by $15 \mathrm{~nm}$ of $\mathrm{Au}$. It was found that the best performance results from $80 \mathrm{~nm} \mathrm{Ag}$. Moreover, in combination 8, $40 \mathrm{~nm}$ of $\mathrm{Ag}$ was deposited on $20 \mathrm{~nm} \mathrm{Cr}$, followed by $15 \mathrm{~nm}$ of $\mathrm{Au}$ in order to further improve uniformity of the films. As expected, $20 \mathrm{~nm}$ Cr resulted in a higher reflectance value.

It can be concluded that the best performance of Ag films was obtained from 80 $\mathrm{nm}$, and the optimal reflectance values were obtained from $20 \mathrm{~nm}$ Cr. Therefore, $80 \mathrm{~nm} \mathrm{Ag}$ was deposited on $20 \mathrm{~nm}$ of $\mathrm{Cr}$, and followed by $15 \mathrm{~nm}$ of $\mathrm{SiO}_{2}$, resulting in a $95 \%$ reflectance value. $93 \%$ reflective mirrors were obtained from $70 \mathrm{~nm} \mathrm{Ag}$. Combinations 11 and 12 were used in this study as the partially reflective and the totally reflective mirrors, respectively.

\subsubsection{X-ray photoelectron spectroscopy}

XPS was used to investigate the presence of silver oxide on the thin film surfaces of unprotected $\mathrm{Ag}$, unprotected and plasma treated $\mathrm{Ag}$, and plasma treated $\mathrm{SiO}_{2}$ 
protected Ag. The films were exposed to oxygen plasma as the real mirror films undergo exposure during plasma bonding the PDMS devices. XPS was carried out for four consecutive days to track the oxidation of the mirrors. Figure 4.5 shows the percent oxidation of the three combinations with time. The unprotected silver film which was not treated with oxygen plasma showed $0.82 \%$ oxidation on the first day, and its oxidation increased and eventually stabilized around $3.1 \%$ on the fourth day.

However, treating the silver film with oxygen plasma increased the oxidation on the film, and the percent oxidation did not stabilize. This trend was expected as oxygen plasma bombards the silver surface and increases the number of defects on the surface, thus increasing the surface energy and allowing more oxidation. Depositing a $\mathrm{SiO}_{2}$ layer successfully protected the silver film as shown in Figure 4.5 , as no oxidation was evident on the surface.

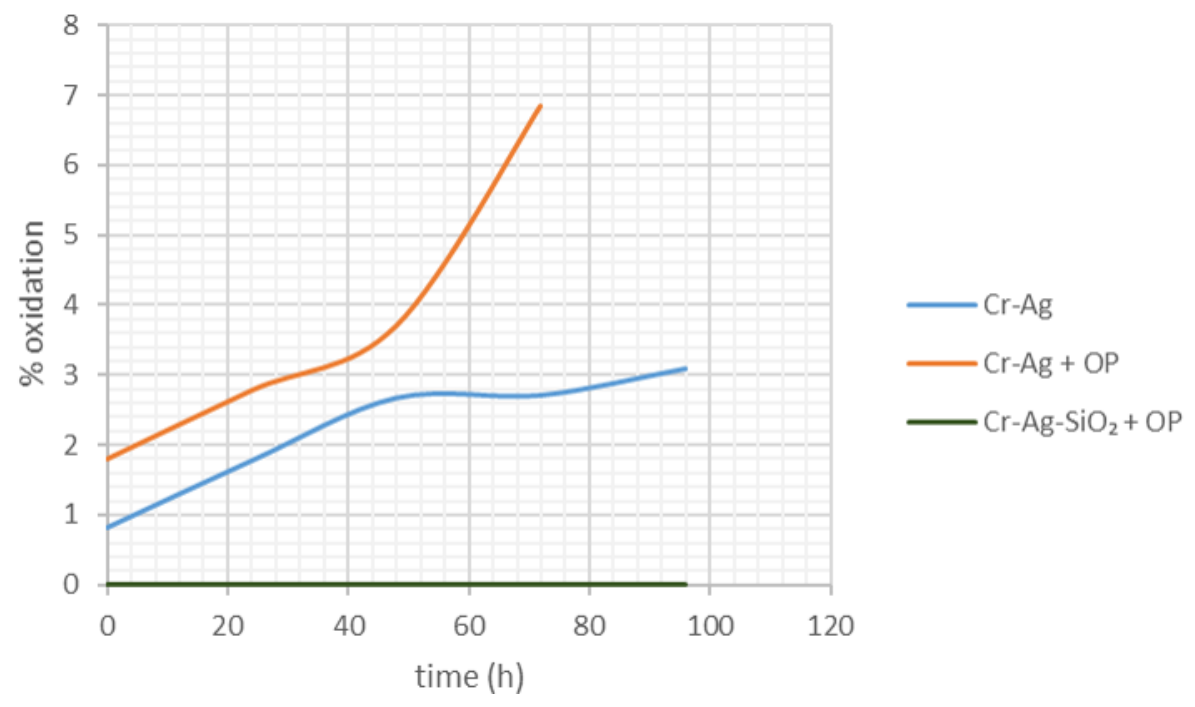

Figure 4.5: \%oxidation over a period of $72 \mathrm{~h}$ for bare silver mirrors exposed and unexposed to oxygen plasma, and for $\mathrm{SiO}_{2}$ protected silver films exposed to oxygen plasma.

The performances of the chosen thin film combinations rely on the presence of the $\mathrm{SiO}_{2}$ film as an oxidation protection layer. The XPS results for the protected and unprotected silver films exposed to oxygen plasma are outlined in Figure 4.6, where Figures 4.6a-c show the oxidation states of the protected mirror surface, 
and Figures 4.6d-e show the oxidation states of the bare Ag mirror surface.
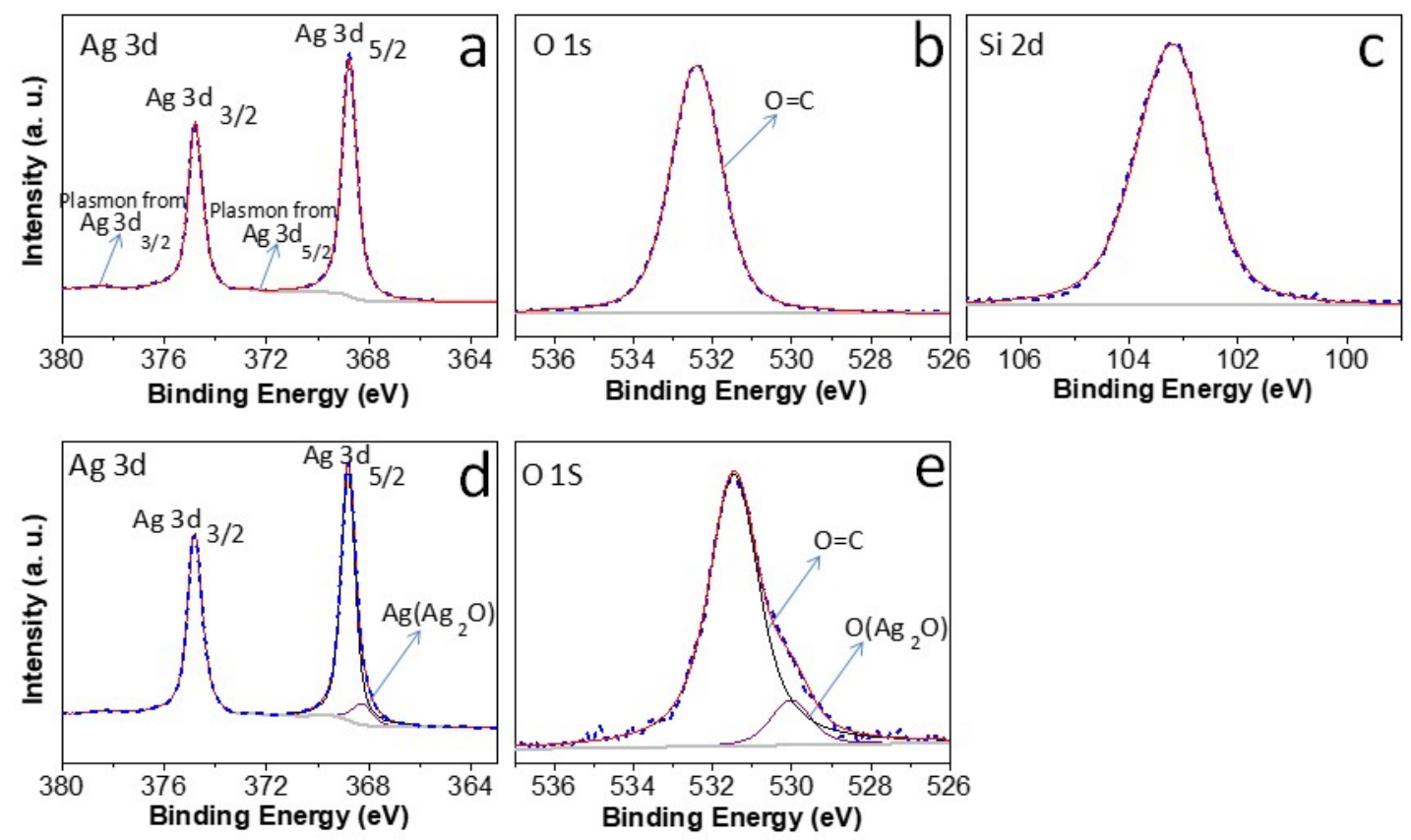

Figure 4.6: (a-c) XPS spectra of silver mirrors exposed to oxygen plasma with oxidation protection layer. (d,e) XPS spectra of bare silver mirrors exposed to oxygen plasma. (a, d) Show Ag 3d spectra, (b,e) show the O 1s spectra, and (c) shows the Si $2 p$ spectrum.

The silver spectrum shown in Figure 4.6a indicates that silver existed $\operatorname{as~} \mathrm{Ag}^{0}$ on the protected mirror surface. The $\mathrm{Ag} 3 \mathrm{~d}_{5 / 2}$ and $\mathrm{Ag} 3 \mathrm{~d}_{3 / 2}$ peaks existed at binding energies of of $368.8 \mathrm{eV}$ and $374.8 \mathrm{eV}$, respectively. Their satellite peaks were also evident at 372.6 and $378.6 \mathrm{eV}$ respectively [52]. In the oxygen spectrum shown in Figure 4.6b, the $\mathrm{O}$ 1s characteristic peak was found at $532.5 \mathrm{eV}$. This peak was associated with the oxygen present in carbonate species and water vapor adsorbed on the surface, and to the lattice $\mathrm{O}_{2}{ }^{-}$of the $\mathrm{SiO}_{2}$ layer. The XPS spectrum of $\mathrm{Si}$ $2 \mathrm{~d}$ at a peak position of $103.3 \mathrm{eV}$ shown in Figure 4.6c confirmed Si presence as $\mathrm{SiO}_{2}$ on the surface of the mirror film.

The XPS peaks of the unprotected mirrors are presented in Figures 4.6d-e. The peak positions at 368.8 and $368.2 \mathrm{eV}$, along with the disappearance of the satellite peaks in the $\mathrm{Ag} 3 \mathrm{~d}$ spectrum (Figure 4.6d) indicated the presence of 
$\mathrm{Ag}$ and $\mathrm{Ag}_{2} \mathrm{O}$. The presence of $\mathrm{AgO}$ was not confirmed nor rejected due to its instability in ultra-high vacuum conditions. A peak at $529.9 \mathrm{eV}$ in the $\mathrm{O} 1 \mathrm{~s}$ spectrum (Figure 4.6e) confirmed the formation of an oxide layer $\mathrm{Ag}_{2} \mathrm{O}$ on the silver films.

The $\mathrm{O}$ 1s peak at 531.5 could be associated with oxygen present in carbonate and water vapor species adsorbed on the surface, or to $\mathrm{Ag}$-O-Ag bonds [53]. In conclusion, the deposition of $\mathrm{SiO}_{2}$ as a protection layer prevented the formation of silver oxide and resulted in mirrors with the highest reflectance values.

\subsubsection{Roughness and SEM}

The XPS and reflectance results for the mirrors used in the FP cavity (combinations 11 and 12) imply the smoothness and uniformity of the mirror films. An SEM image was obtained for the mirror surface as shown in Figure 4.7, where a crack free surface was observed as shown in the inset. Moreover, AFM was also used to quantify the mirror roughness, which was found to be $0.7 \pm 0.1 \mathrm{~nm}$.

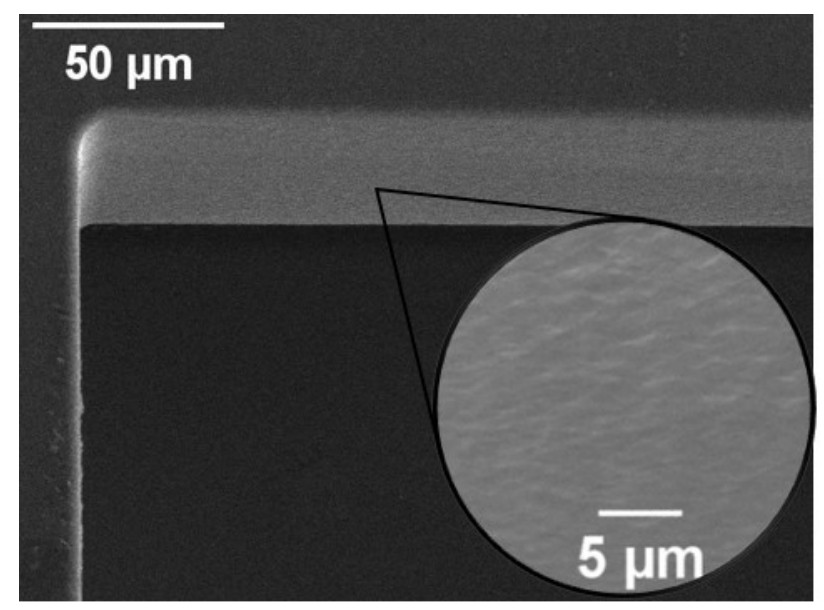

Figure 4.7: SEM image of the deposited mirror. 


\subsubsection{Optical gain measurements}

The optical gain of $\mathrm{Cd}_{1-x} \mathrm{Zn}_{x} \mathrm{~S}$ core/crown@shell CQWs in toluene, and CdSe/CdS@ZnS core/crown@ 4ML shell CQWs in water were studied in this section. The absorption and photoluminescence (PL) spectra of the CQWs used are shown in Figures 4.2a,c. Lasing and ASE of $\mathrm{Cd}_{1-x} \mathrm{Zn}_{x} \mathrm{~S}$ core/crown@shell CQWs were observed in capillary, and ASE of CdSe/CdS@ZnS core/crown@ 4ML shell CQWs was observed in flow. The $4 \mathrm{~mm}$ channels used in these experiments had a width and height of 300 and $100 \mu \mathrm{m}$, respectively. The pump laser used in this study was a $400 \mathrm{~nm}$ laser beam with 120 fs pulse width and $1 \mathrm{kHz}$ repetition rate, and the samples were excited using one-photon absorption.

\subsubsection{Capillary operation}

To evaluate the optical gain of $\mathrm{Cd}_{1-x} \mathrm{Zn}_{x} \mathrm{~S}$ core/crown@shell CQWs in microchannels, mirrorless devices were filled with CQWs using capillary forces. In order to prevent drying, a pipette tip was placed on the channel inlet and was filled with a solution of CQWs. The operating conditions for lasing were similar to those used for the optical gain measurements. The devices used for lasing,

however, had mirrors deposited on their channel walls as described in Section 3.2.1.

At pump intensities below $17 \mu \mathrm{J} / \mathrm{cm}^{2}$, spontaneous emission centered at 637.5 $\mathrm{nm}$ with an emission linewidth of $29.2 \mathrm{~nm}$ was observed from the devices. At a pump intensity of $17.1 \mu \mathrm{J} / \mathrm{cm}^{2}$, the emergence of a sharp peak centered at 648.1 $\mathrm{nm}$ with an emission linewidth of $6.8 \mathrm{~nm}$ was observed, marking the transition of the emission behavior from spontaneous emission (SE) to amplified spontaneous emission (ASE).

Beyond this pump intensity, the ASE emission spectrum was dominant, and further increasing the pump intensity increased the output intensity of the system. The optical emission of the device at different input pump intensity values, and the change of the total output intensity and the FWHM of the system with 

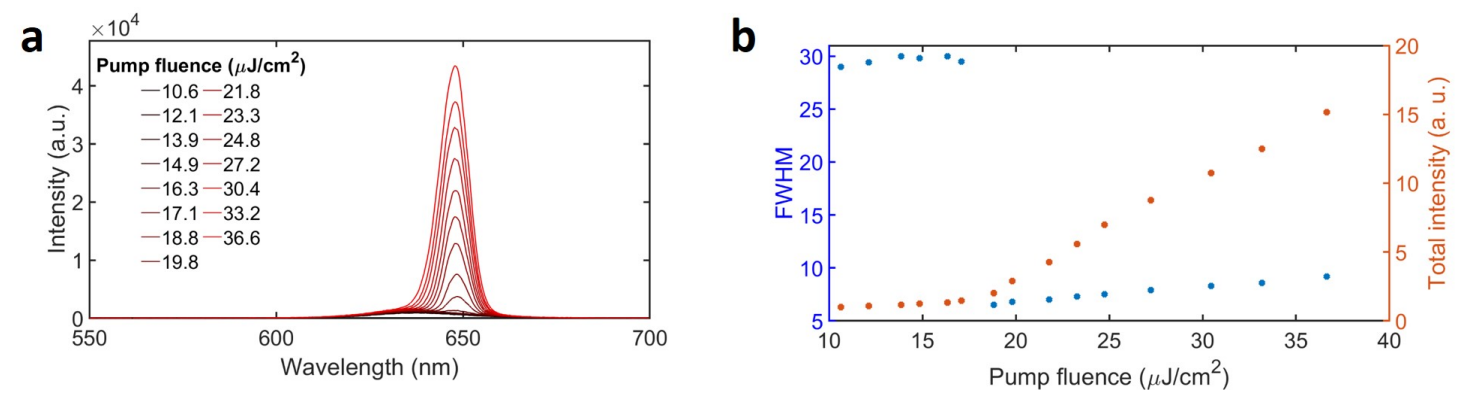

Figure 4.8: (a) Photoluminescence of $\mathrm{Cd}_{1-x} \mathrm{Zn}_{x} \mathrm{~S}$ core/crown@shell CQWs in capillary operation at increasing pump intensities. (b) Pump fluence vs linewidth and output intensity.

increasing pump intensity were plotted as shown in Figure 4.8a, and Figure 4.8b, respectively.

Spontaneous emission dominated the spectrum at low pump intensities, and its characteristics were similar to the SE observed prior to the onset of ASE in the optical gain measurement experiment. At a pump intensity of $68.4 \mu \mathrm{J} / \mathrm{cm}^{2}$, a $0.54 \mathrm{~nm}$ narrow emission peak emerged, and further increasing the input pump intensity resulted in an increase in the output intensity. This behavior marked the transition from SE to lasing. The output laser beam focused onto a screen is shown in Figure 4.9c.

The emission linewidth of the device dropped from $27 \mathrm{~nm}$ centered at $642.6 \mathrm{~nm}$ at spontaneous emission to $0.54 \mathrm{~nm}$ centered around $645.6 \mathrm{~nm}$ during lasing. The laser emission saturated at pump intensities of $76.1 \mu \mathrm{J} / \mathrm{cm}^{2}$ and above. The device emission with increasing pump intensities was collected and is shown in Figure 4.9a. The drop in the linewidth values of the emission as well as the increase in the output intensity were plotted as shown in Figure 4.9b. The expected "S"-shaped behavior of the laser beam is shown in Figure 4.9b.

\subsubsection{Pressure-driven operation}

In this section, the optical gain of the aqueous CdSe/CdS@ZnS core/crown@ 4ML shell CQWs in flow was evaluated. Water was introduced to the channels as the 
a

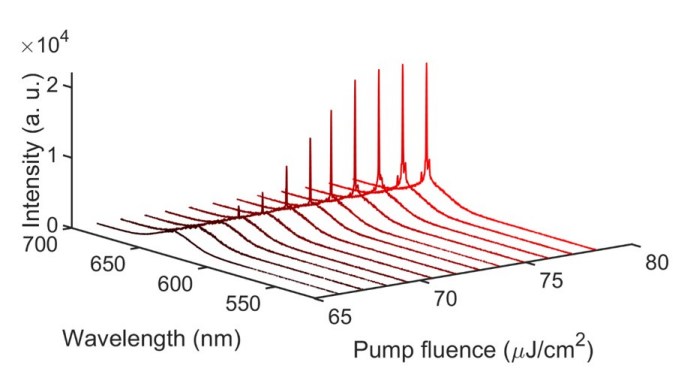

b

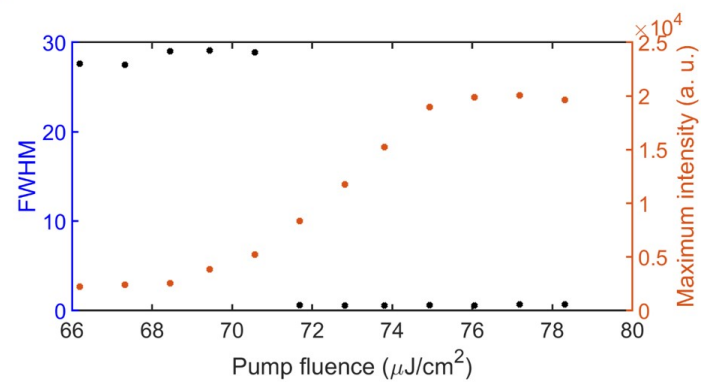

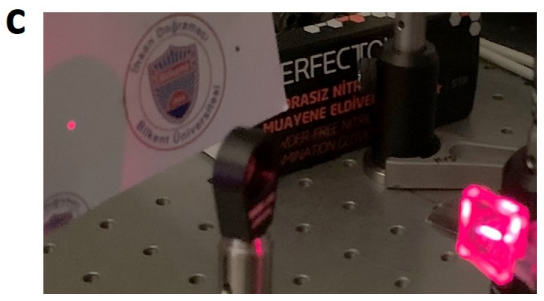

Figure 4.9: (a) Lasing of $\mathrm{Cd}_{1-x} \mathrm{Zn}_{x} \mathrm{~S}$ core/crown@shell CQWs in capillary. (b) Linewidth and luminescence of the output laser beam vs input pump intensity. (c) Image of the output laser beam.

cladding, and the CQWs solution was introduced as the core fluid as described in Section 2.3.2.2, using syringe pumps. The flowrates of water and CQWs solution used were 1 and $4 \mu \mathrm{L} / \mathrm{min}$, respectively, and the width of the channel occupied by CQWs was $240 \mu \mathrm{m}$ according to Equation 2.24. The optical set-up used for these experiments was identical to the one used for optical gain measurements in capillary.

Spontaneous emission with a linewidth of $25.2 \mathrm{~nm}$ centered at $644.1 \mathrm{~nm}$ was observed from these CQWs in flow at low pump intensities. The emergence of a narrow peak in Figure 4.10a accompanied with a sharp increase in the total output intensity (Figure 4.10b) was observed at a pump intensity of $468.5 \mu \mathrm{J} / \mathrm{cm}^{2}$. This behavior marked the transition to ASE, and the narrow peak had a linewidth of $9.8 \mathrm{~nm}$ and was centered at $634.5 \mathrm{~nm}$. 

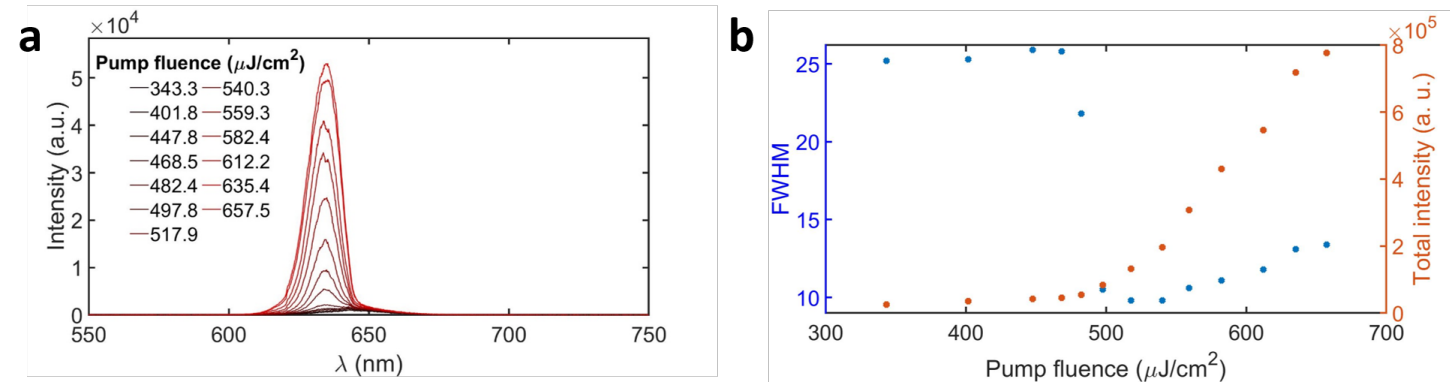

Figure 4.10: (a) Photoluminescence spectra of 4 ML CdSe/CdS@ZnS core/crown@ 4ML shell CQWs in flow various pump intensities. (b) The change in luminescence and linewidth with increasing pump intensities. 


\section{Chapter 5}

\section{Conclusion and future outlook}

In summary, this thesis work investigated light amplification and lasing from organic and aqueous solutions of CQWs in PDMS microfluidic platforms. Waveguides were created in microfluidic channels to achieve optical gain, and the CQWs were introduced to the channels in two mechanisms, namely capillary filling and pressure-driven flow.

Previous works on microfluidic lasers report lasing thresholds of $1.1 \mathrm{~mJ} / \mathrm{cm}^{2}$ and $2.6 \mathrm{~mJ} / \mathrm{cm}^{2}$ for multimode and single-mode lasing, with the residence times of the dye solutions in these channels varying from 60 to $360 \mathrm{~ms}$ to prevent photobleaching. By employing CQWs as the gain material in this work, we were able to address the problems of high thresholds and high flow rates associated with dye lasers. The CQWs used in this work show gain in static solutions at the power levels tested (upto $700 \mu \mathrm{J} / \mathrm{cm}^{2}$ ) with no observable photobleaching. This offers the advantage of observing optical gain and lasing from CQWs using the minimum amounts of gain material. A few microliters of solutions of CQWs were used for the capillary filled devices, where the gain material was not replenished during operation. Moreover, a single-mode lasing threshold lower by two orders of magnitude compared to the reported microfluidic lasers with a value of 17 $\mu \mathrm{J} / \mathrm{cm}^{2}$ was realized from a capillary microfluidic CQW laser. 
In capillary operation, a solution of CQWs in toluene was used as the gain medium, and the PDMS was used as the cladding material for the waveguide. In pressure-driven operation, a liquid-liquid waveguide was created, where a solution of CQWs in water was used as the gain material, and a soft wall of water created by flow focusing was used as the cladding material. Moreover, FP cavities were created by the depositing mirrors on the channel walls.

Optical gain of an aqueous solution of $4 \mathrm{ML}$ CdSe/CdS@ 4 ML CdZnS core/crown@shell CQWs in a PDMS based laminar liquid-liquid waveguide was demonstrated in pressure-driven operation. To our knowledge, this is the first demonstration of in-solution ASE from water-based CQWs, and ASE from CQWs in flow in microfluidic platforms. This method of operation allows for the adjustment of the waveguide dimensions during operation, which is otherwise not possible in capillary operation.

A single-mode laser with an ultra-low threshold was demonstrated in capillary using a solution of $4 \mathrm{ML} \mathrm{CdSe} / \mathrm{CdS} @ 3 \mathrm{ML} \mathrm{Cd}_{1-x} \mathrm{Zn}_{x} \mathrm{~S}$ core/crown@shell CQWs as the gain medium. The introduction of the CQWs to the channel by capillary action eliminated the need for external syringe pumps, and offered the advantage of ease of operation. A user friendly, in-solution laser was achieved thanks to the CQWs solution's photo-stability and strong capillary interaction with PDMS. To our knowledge, this is the first demonstration of single-mode lasing from CQWs in solution.

On the expense of lack of tunability, capillary driven microfluidic lasers allow for the realization of ultra-low threshold lasers using a minimal amount of the gain material. The PDMS based microfluidic devices may be redesigned to hold more than one channel to be filled with different CQWs emitting in various colors, providing a good starting point for the development of in-solution, user friendly, low-threshold multicolor lasers.

We believe that our pressure-driven microfluidic waveguides provide a promising platform for the realization of multi-color laminar-flow lasers, which enable the user to tune the output laser beam while operating. Finally, the findings of 
this work can be further extended to create in-solution broadband light sources which cover the visible light spectrum. 


\section{Bibliography}

[1] D. V. V. et al., "A low-threshold, high-efficiency microfluidic waveguide laser," Journal of the American Chemical Society, vol. 127, no. 25, pp. 89528953, 2005.

[2] S. T. et al., "A multi-color fast-switching microfluidic droplet dye laser," Lab on a Chip, vol. 9, no. 19, pp. 2767 - 2771, 2009.

[3] S. Tang, R. Derda, Q. Quan, M. Lončar, and G. Whitesides, "Continuously tunable microdroplet-laser in a microfluidic channel," Optics Express, vol. 19, no. 3, pp. $2204-2215,2011$.

[4] Y. Kong, H. Dai, X. He, Y. Zheng, and X. Chen, "Reconfigurable rgb dye lasers based on the laminar flow control in an optofluidic chip," Optics Letters, vol. 43, no. 18, pp. 4461-4464, 2018.

[5] C. Eggeling, J. Widengren, R. Rigler, and C. A. M. Seidel, "Photobleaching of fluorescent dyes under conditions used for single-molecule detection: Evidence of two-step photolysis," Analytical Chemistry, vol. 70, no. 13, pp. 2651$2659,1998$.

[6] R. Zondervan, F. Kulzer, M. A. Kol'chenk, and M. Orrit, "Photobleaching of rhodamine $6 \mathrm{~g}$ in poly (vinyl alcohol) at the ensemble and single-molecule levels," J. Phys. Chem. A, vol. 108, pp. 1657-1665, 2004.

[7] N. Tessler, V. Medvedev, M. Kazes, S. H. Kan, and U. Baninl, "Efficient nearinfrared polymer nanocrystat light-emitting diodes," Science, vol. 295, pp. 1506-1508, 2002. 
[8] V. I. Klimov, A. A. Mikhailovsky, S. Xu, A. V. Malko, J. A. Hollingsworth, C. A. Leatherdale, H.-J. Eisler, and M. G. Bawendi, "Optical gain and stimulated emission in nanocrystal quantum dots," Science, vol. 290, pp. 314-317, 2000.

[9] R. D. Schaller and V. I. Klimov, "High efficiency carrier multiplication in pbse nanocrystals: Implications for solar energy conversion," Phys. Rev. Lett., vol. 92, p. 186601, 2004.

[10] A. P. Alivisatos, "The use of nanocrystals in biological detection," Nature Biotechnol., vol. 22, pp. 47-52, 2004.

[11] Baghani and E. et al., "Auger-limited carrier recombination and relaxation in cdse colloidal quantum wells," The Journal of Physical Chemistry Letters, vol. 6.6, pp. 1032-1036, 2015.

[12] S. Ithurria, M. D. Tessier, B. Mahler, R. P. S. M. L. abd B. Dubertret, and A. L. Efros, "Colloidal nanoplatelets with two-dimensional electronic structure," Nature Materials, vol. 10, no. 12, pp. 936-941, 2011.

[13] B. Guzelturk, M. Pelton, M. Olutas, and H. V. Demir, "Giant modal gain coefficients in colloidal ii-vi nanoplatelets," Nano Letters, vol. 19, no. 1, pp. 277-282, 2018.

[14] M. D. Tessier, M. D. C. Javaux, I. Maksimovic, V. Loriette, and B. Dubertret, "Spectroscopy of single cdse nanoplatelets," ACS Nano, vol. 6, no. 8, pp. 6751-6758, 2012.

[15] E. S. Bahaa and C. T. Malvin, Fundamentals of photonics. John Wiley and sons, 1991.

[16] C. P. Bergmann and F. Machado, Carbon nanomaterials as adsorbents for environmental and biological applications. New York: Springer International Publishing, 2015.

[17] S. Gaponenko and H. Demir, "Quantum confinement effects in semiconductors," Applied Nanophotonics, pp. 52-91, 2018. 
[18] N. W. Ashcroft and N. D. Mermin, Solid State Physics. NNY: Brooks Cole., 1976.

[19] W. Cao, Semiconductor Photocatalysis: Materials, Mechanisms and Applications. BoD-Books on Demand, 2016.

[20] Y. Kelestemur, B. Guzelturk, O. Erdem, M. Olutas, K. Gungor, and H. V. Demir, "Platelet-in-box colloidal quantum wells: Cdse/cds@ cds core/crown@ shell heteronanoplatelets.," Advanced Functional Materials, vol. 26, no. 21, pp. 3570-3579, 2016.

[21] Y. Zhao, "Quantum dots and doped nanocrystals: Synthesis, optical properties and bio-applications," Doctoral dissertation, Utrecht University, pp. 13,14, 2013.

[22] Z. Zhao, M. A. Carpenter, and M. A. Petrukhina, Semiconductor Gas Sensors: Semiconductor quantum dots for photoluminescence-based gas sensing. Woodhead Publishing, 2013.

[23] R. Bird, W. Stewart, and E. Lightfoot, Transport Phenomena. New York: Wiley, 2002.

[24] B. E. Rapp, Microfluidics: modeling, mechanics and mathematics. William Andrew Publishing, 2016.

[25] D. Wibowo, C. X. Zhao, and Y. He, In Microfluidics for Pharmaceutical Applications: Fluid properties and hydrodynamics of microfluidic systems. William Andrew Publishing, 2019.

[26] T. Bergman, Introduction to heat transfer. USA: Wiley, 2015.

[27] H. Bruus, Theoretical microfluidics. Oxford: Oxford university press, 2008.

[28] G. B. Lee, C. C. Chang, S. B. Huang, and R. J. Yang, "The hydrodynamic focusing effect inside rectangular microchannels.," Journal of Micromechanics and Microengineering, vol. 16, no. 5, p. 1024, 2006. 
[29] J. B. Knight, A. Vishwanath, J. P. Brody, and R. H. Austin, "Hydrodynamic focusing on a silicon chip: mixing nanoliters in microseconds.," Physical Eeview Letters, vol. 80, no. 17, p. 3863, 1998.

[30] A. Olanrewaju, M. Beaugrand, M. Yafia, and D. Juncker, "Capillary microfluidics in microchannels: from microfluidic networks to capillaric circuits.," Lab on a Chip, vol. 18, no. 16, pp. 2323-2347, 2018.

[31] D. M. Hernández, Advanced Optical Instruments and Techniques. CRC Press, 2017.

[32] J. M. Quero, F. Perdigones, and C. Aracil, Smart Sensors and MEMS. Woodhead Publishing, 2018.

[33] M. J. Madou, Fundamentals of microfabrication: the science of miniaturization. CRC Press, 2002.

[34] S. P. Lacour and T. Someya, Stretchable Electronics. John Wiley and Sons, 2012.

[35] O. Graudejus, P. Gorrn, and S. Wagner, "Controlling the morphology of gold films on poly (dimethylsiloxane).," ACS Appl. Mater. Interfaces, vol. 2, no. 7, pp. 1927-1933, 2010.

[36] K. Wasa, M. Kitabatake, and H. Adachi, Thin film materials technology: sputtering of control compound materials. Springer Science and Business Media, 2004.

[37] G. Hass, "Reflectance and preparation of front-surface mirrors for use at various angles of incidence from the ultraviolet to the far infrared." JOSA, vol. 72 , no. 1 , pp. 27-39, 1982.

[38] J. B. Heaney, L. R. Kauder, S. C. Freese, and M. A. Quijada, "”preferred mirror coatings for uv, visible, and ir space optical instruments." in earth observing systems xvi," International Society for Optics and Photonics, vol. 8510, p. $85100 \mathrm{~F}, 2012$. 
[39] G. Hass, J. B. Heaney, H. Herzig, J. F. Osantowski, and J. J. Triolo., "Reflectance and durability of ag mirrors coated with thin layers of al 2 o 3 plus reactively deposited silicon oxide.," Applied Optics, vol. 14, no. 11, pp. 2639 $2644,1975$.

[40] S. H. Ali, "Advanced nanomeasuring techniques for surface characterization," International Scholarly Research Notices, vol. 2012, 2012.

[41] H. Konno, Materials Science and Engineering of Carbon. ButterworthHeinemann, 2016.

[42] E. Constable, Comprehensive coordination chemistry II: from biology to nanotechnology. Newnes, 2003.

[43] A. Piegari and F. Flory, Optical thin films and coatings: From materials to applications. Woodhead Publishing, 2018.

[44] R. Shahbazian-Yassar, Q. J. Wang, and Y. Chung, Atomic Force Microscopy in Encyclopedia of Tribology. Springer, Boston, MA, 2013.

[45] J. N. Lee, C. Park, and G. M. Whitesides, "Solvent compatibility of poly (dimethylsiloxane)-based microfluidic devices.," Analytical Chemistry, vol. 75, no. 23, pp. 6544-6554, 2003.

[46] Y. Y. Huang, K. Choi, and C. H. Hidrovo, "The improved resistance of pdms to pressure-induced deformation and chemical solvent swelling for microfluidic devices.," Microelectronic Engineering, vol. 124, pp. 66-75, 2014.

[47] D. Fuard, T. Tzvetkova-Chevolleau, S. Decossas, P. Tracqui, and P. Schiavone, "Optimization of poly-di-methyl-siloxane (pdms) substrates for studying cellular adhesion and motility.," Microelectronic Engineering, vol. 124, no. 85, p. 1289-1293, 2008.

[48] J. Y. Park, S. J. Yoo, E.-J. Lee, D. H. Lee, J. Y. Kim, and S.-H. Lee, "Increased poly (dimethylsiloxane) stiffness improves viability and morphology of mouse fibroblast cells," BioChip Journal, vol. 4, no. 3, pp. 230-236, 2010.

[49] A. Belkind and S. Gershman, "Plasma cleaning of surfaces.," Vacuum Coating and Technology November, pp. 46-57, 2008. 
[50] B. J. Inkson, "Scanning electron microscopy (SEM) and transmission electron microscopy (TEM) for materials characterization." Materials characterization using nondestructive evaluation (NDE) methods. Woodhead Publishing, 2016.

[51] S. Shendre, S. Delikanli, M. Li, D. Dede, Z. Pan, S. T. Ha, Y. H. Fu, P. L. Hernández-Martínez, O. Erdem, and A. I. Kuznetsov, "Ultrahigh-efficiency aqueous flat nanocrystals of cdse/cds@ $c d_{1-x} z n_{x} s$ colloidal core/crown@ alloyed-shell quantum wells.," Biochip Journal, vol. 11, no. 1, pp. 301-310, 2019.

[52] J. Feng, D. Fan, Q. Wang, L. Ma, W. Wei, J. Xie, and J. Zhu, Colloids and Surfaces A: Physicochem.Eng.Aspects. Facile synthesis silver nanoparticles on different xerogel supports as highly efficient catalysts for the reduction of p-nitrophenol. Elsevier, 2017.

[53] N. J. Firet, M. A. Blommaert, T. Burdyny, A. Venugopal, D. Bohra, A. Longo, and W. A. Smith, "Operando exafs study reveals presence of oxygen in oxide-derived silver catalysts for electrochemical $\mathrm{co}_{2}$ reduction.," Journal of Materials Chemistry A, vol. 7, no. 6, pp. 2597-2607, 2019. 


\section{Appendix A}

\section{Photolithography parameters}

\begin{tabular}{|c|c|c|c|}
\hline \multicolumn{4}{|c|}{ Spin coating parameters } \\
\hline & Velocity (rpm) & Acceleration $(\mathrm{rpm} / \mathrm{s})$ & Time (s) \\
\hline \multicolumn{4}{|c|}{ Base layer } \\
\hline Step 1 & 500 & 100 & 25 \\
\hline Step 2 & 2500 & 200 & 40 \\
\hline \multicolumn{4}{|c|}{ Main layer } \\
\hline Step 1 & 500 & 50 & 40 \\
\hline Step 2 & 2200 & 300 & 35 \\
\hline
\end{tabular}

\begin{tabular}{|l|l|l|}
\hline \multicolumn{3}{|c|}{ Pre-exposure bake parameters } \\
\hline \multicolumn{2}{|c|}{ Temperature $\left({ }^{\circ} \mathrm{C}\right)$} & Time (minutes) \\
\hline Base layer \\
\hline Step 1 & 65 & 2 \\
\hline Step 2 & 95 & 4 \\
\hline Step 3 & 65 & 1 \\
\hline Main layer \\
\hline Step 1 & 65 & 3 \\
\hline Step 2 & 95 & 8 \\
\hline Step 3 & 65 & 2 \\
\hline
\end{tabular}




\begin{tabular}{|c|c|c|}
\hline Mask aligner parameters & \multicolumn{3}{|l|}{ Base } & Main \\
\hline Layer & Manual top side & Manual top side \\
\hline Process & Soft contact & Soft contact \\
\hline Contact mode & 100 & 200 \\
\hline Separation $(\mu m)$ & 2.3 & 2.3 \\
\hline Mask thickness $(\mathrm{mm})$ & 0.5 & 0.5 \\
\hline Sample thickness $(\mathrm{mm})$ & 2 & 100 \\
\hline Resist thickness $(\mu \mathrm{m})$ & 120 & 120 \\
\hline Exposure intensity $\left(\mathrm{mJ} / \mathrm{cm}^{2}\right)$ & &
\end{tabular}

\begin{tabular}{|c|c|c|}
\hline \multicolumn{3}{|c|}{ Post-exposure bake parameters } \\
\hline & Temperature $\left({ }^{\circ} \mathrm{C}\right)$ & Time (minutes) \\
\hline \multicolumn{3}{|c|}{ Base layer } \\
\hline Step 1 & 65 & 1 \\
\hline Step 2 & 95 & 3 \\
\hline Step 3 & 65 & 1 \\
\hline \multicolumn{3}{|c|}{ Main layer } \\
\hline Step 1 & 65 & 3 \\
\hline Step 2 & 95 & 8 \\
\hline Step 3 & 65 & 2 \\
\hline
\end{tabular}

